\title{
The Assessment of Potential Observability for Joint Chemical States and Emissions in Atmospheric Modelings
}

\section{Xueran Wu ( $\boldsymbol{\nabla}$ x.wu@fz-juelich.de)}

Forschungszentrum Julich GmbH https://orcid.org/0000-0003-1003-3693

Hendrik Elbern

Forschungszentrum Julich $\mathrm{GmbH}$

Birgit Jacob

Bergische Universitat Wuppertal

\section{Research Article}

Keywords: data assimilation, Kalman smoother, singular value decomposition, the degree of freedom for signal, observability

Posted Date: August 3rd, 2021

DOl: https://doi.org/10.21203/rs.3.rs-750110/v1

License: (c) (i) This work is licensed under a Creative Commons Attribution 4.0 International License. Read Full License 


\title{
The assessment of potential observability for joint chemical states and emissions in atmospheric modelings
}

\author{
Xueran Wu • Hendrik Elbern · Birgit \\ Jacob
}

Received: date / Accepted: date

\begin{abstract}
In predictive geophysical model systems, uncertain initial values and model parameters jointly influence the temporal evolution of the system. As for chemistry-transport models, emission rates are at least as important as initial values for model evolution controls. This renders initial-value-only optimization by traditional data assimilation methods as insufficient. However, blindly extending the optimization parameter set jeopardizes the validity of the resulting analysis since the ill-posedness of the inversion task is increased. Hence, it becomes important to assess the potential observability of measurement networks for model state and parameters in atmospheric modelings in advance of the optimization. In this paper, we introduce an approach to quantify the impact of observation networks jointly for initial trace gas state and emission rates for transport-diffusion models extended by emissions. Applying a Kalman smoother as underlying assimilation technique, we develop a quantitative assessment method to evaluate the potential observability and the sensitivity of observation networks to initial values and emission rates. For practical applications, we derive an ensemble based version of the approach and give several elementary experiments for illustration.
\end{abstract}

\footnotetext{
Xueran $\mathrm{Wu}$

IEK-8, Forschungszentrum Jülich, Wilhelm-Johnen-Straße,52428, Jülich, Germany

Rhenish Institute for Environmental Research at the University of Cologne, Pohligstr. 3, 50969, Cologne, Germany

Mathematics Department, Unversity of Wuppertal, Gaußstraße 20, 42119, Wuppertal, Ger-

many

E-mail: x.wu@fz-juelich.de

Hendrik Elbern

IEK-8, Forschungszentrum Jülich, Wilhelm-Johnen-Straße,52428, Jülich, Germany Rhenish Institute for Environmental Research at the University of Cologne, Pohligstr. 3, 50969, Cologne, Germany

Birgit Jacob

Mathematics Department, Unversity of Wuppertal, Gaußstraße 20, 42119, Wuppertal, Germany
} 
Keywords data assimilation - Kalman smoother · singular value decomposition · the degree of freedom for signal · observability

\section{Introduction}

Climate change and air quality are influenced by fluxes of green house gases, reactive gas emissions and aerosols. The temporal evolution of reactive chemistry in the atmosphere is usually modeled by atmospheric chemistry transport models. Poorly known initial values, sources and sinks cause a serious problem for the quality of simulation, which is addressed by data assimilation and inverse modeling. In practice data assimilation problems are typically solved in the situation where the number of observations are markedly lower than the model degree of freedom [14]. Consequently, when aiming to improve the quality of data assimilation and inverse modeling results, several aspects can be considered. These include (i) to optimize the observation network, subject to given external constraints, (ii) to evaluate the value of individual or types of observations for the analysis, and (iii) to quantify the degree of which the analysis can be influenced by the observations, which is related to the sensitivity.

The observation network optimization problem (i) has been addressed traditionally by Observation System Simulation Experiments (OSSEs, e.g [14]). The advanced concept of targeted observations has been popularized during the FASTEX campaign (e.g [28], [43]). Theoretical studies are presented, for example by [3], or recently by [2] for a case of study of highly nonlinear dynamics, and [46] for the optimal deployment of observations for time-varying system in a infinite dimensional domain within a finite-time interval. The problem (ii) addressing the benefit assessment of individual observations or types of measurements has been investigated by [10] and a sequence of related papers. Finally, the need to quantify the information content provided by the observations (iii) can be satisfied by suitably selected measures. Singular value decomposition (SVD) is a well-known tool applied to identify the priorities of observations by detecting the fastest growing uncertainties in meteorological models (e.g [9], [25], [26], [27], [31], [32], [39],[40], [42]). [6] investigated methods to approximate the solutions of high-dimensional linear Bayesian problems and used a scalable randomized SVD algorithm to solve a maximum problem of the information content of the Bayesian inversion. Besides, the concept of DFS has been frequently applied to satellite retrieval problems, typically of significantly lower dimensions when compared to data assimilation (see for example [19], [20], [33], [37], [38]). In this context a meanwhile classical task is greenhouse gas inversion, from which a rich set of literature emerged (e.g [36]). In these studies, the sensitivity of the model evolution with respect to the model errors and the observation network is a key quantity to be analyzed. Several methodologies have been proposed to account for model errors in both variational and ensemble data assimilation (e.g. [2], [13], [29], [41], [49]). In [35], the authors outlined the perceptibility and stability in optimal param- 
eter estimation in meteorology and oceanography. [12] introduced a general framework to optimize a set of parameters controlling the 4D-var data assimilation system. In a related paper [11], the authors applied it into shallow water model state and other parameters .

Most studies cited above were motivated in support of the classical data assimilation problem with initial values or prognostic state variables as the only parameters to be optimized. However, for chemistry transport or greenhouse gas models, which are driven by emissions in the troposphere, the optimization of the initial state is no longer the only issue. In order to get better analysis from combining the model with observations, efforts of joint optimization have been made by adding the emission rates to concentrations in amount of manuscripts, such as [5],[15], [16],[17], [22], [23] [34],[44], [45]. But the lack of ability to observe and estimate surface emission fluxes directly is still a major roadblock hampering the progress in predictive skills of climate and atmospheric chemistry models. Thus, the capacity to distinguish between the degree of freedom for signal of both emission rates and concentrations is crucial to both assess the value of a measurement network and the estimation of emission strength. Therefore, in this paper we investigate an approach to identify the potential observability and sensitivity of any given observation configuration to initial value and emission rates for atmospheric transport diffusion models. With the Kalman smoother as the appropriate data assimilation method, we focus on identifying the impacts and limits of measurement networks to optimize chemical states and emission rates jointly by comparing their relative sensitivities. Meanwhile, the expected results display the geographical distribution for joint initial values and emission rates.

The rest of paper is organized as follows. In section 2, we establish the dynamic model for emissions and extend the original atmospheric transport model by emission rates in a novel way. In section 3, based on the Kalman smoother, we present the specific approach to determine the degree of freedom for signal for both initial values and emission rates. In section 4 , we develop the ensemble approach to evaluate the degree of freedom for signal of the initial value and emission rates based on the non-singularity of the background covariance matrix. In section 5, we identify the sensitive directions of the initial values and emission rates separately through maximize the ratio of the magnitude of observation perturbation and the initial perturbation. It also provides us a possibility to estimate the sensitivities of the initial values and emission rates by few leading singular values and singular vectors. In section 6 , we extend a 3D advection-diffusion equation with the dynamic model of the emission rate and give several elementary experiments to verify and demonstrate the ensemble approach. Finally, in section 7 , we conclude the main contributions of this paper and discuss possible extensions. 
2 Atmospheric inverse modeling extended by emission rates

We usually describe the concentration change rate by the following prognostic atmospheric transport model

$$
\frac{d c(t)}{d t}=\mathcal{A}(c)+e(t)
$$

where $\mathcal{A}$ is a nonlinear model operator, $c(t)$ and $e(t)$ are the state vector of chemical constituents and emission rates at time $t$, respectively .

The prior estimate of the state vector of concentrations $c(t)$ is given and denoted by $c_{b}(t)$, termed as the background state. The prior estimate of emission rates, usually taken from emission inventories, is denoted by $e_{b}(t)$.

Let $\mathbf{A}$ be the tangent linear operator of $\mathcal{A}, \delta c\left(t_{0}\right)=c\left(t_{0}\right)-c_{b}\left(t_{0}\right)$ and $\delta e(t)=e(t)-e_{b}(t)$. The linear evolution of the perturbation of $c(t)$ follows the tangent linear model as

$$
\frac{d \delta c}{d t}=\mathbf{A} \delta c+\delta e(t)
$$

By the discretization of the tangent linear model in space, it is straightforward to obtain the linear solution of (2) discretized in space and continuous in time as

$$
\delta c(t)=M\left(t, t_{0}\right) \delta c\left(t_{0}\right)+\int_{t_{0}}^{t} M(t, s) \delta e(s) d s,
$$

where $M(\cdot, \cdot)$ is the resolvent obtained from the spatial discretization of $\mathbf{A}$. Without loss of generality, we assume $\delta c(t) \in \mathbf{R}^{n}$, $\delta e(t) \in \mathbf{R}^{n}$, where $n$ is the dimension of the partial phase space of concentrations and emission rates. Obviously, $M(\cdot, \cdot) \in \mathbf{R}^{n \times n}$.

In addition, let $y(t)$ be the observation vector of $c(t)$ and define

$$
\delta y(t)=y(t)-\mathcal{H}\left(c_{b}\right)(t),
$$

where $\delta y(t) \in \mathbf{R}^{m(t)}, m(t)$ is the dimension of the phase space of observation configurations at time $t . \mathcal{H}(t)$ is a nonlinear forward observation operator mapping the model space to the observation space. Linearizing the nonlinear operator $\mathcal{H}$ as $H$, we present the observation system as

$$
\delta y(t)=H(t) \delta c(t)+\nu(t)
$$

where the observation error $\nu(t)$ of the Gaussian distribution has zero mean and variance $R(t) \in \mathbf{R}^{m(t) \times m(t)}$.

The Kalman smoother is a recursive estimator to provide the best linear unbiased estimates (BLUE) of the unknown variables with error estimates, using a sequence of observations (e.g [21]). In addition to 4D-Var approaches, Kalman smoothers not only can provide the best linear unbiased estimate by a series of observations over time for the state vector, but also update the forecasting error covariances of that estimate.

It is clear to see that if the initial state of concentrations is the only parameter to be optimized, we can only consider the concentrations as the model 
states and apply the Kalman filter and smoother into the tangent linear model (2) with observations (5) directly. However, in most cases the exact values of emission rates are poorly known and also considered as parameters which need to be optimized. It has been shown by [17] that the diurnal profiles are better known than the exact amplitude of emission rates. Hence, we can only consider the amplitude of the diurnal emission cycle as optimization parameters. Thus, we first reformulate the background evolution of emission rates from time $s$ to $t$ in a dynamic form as an emission model

$$
e_{b}(t)=M_{e}(t, s) e_{b}(s), \quad s \leqslant t
$$

where $e_{b}(\cdot)$ is a $n$-dimensional vector, of which the $i^{\text {th }}$ element is denoted by $e_{b}^{i}(\cdot)$ and $M_{e}(t, s)$ is the scaling diagonal matrix defined as

$$
M_{e}(t, s)=\left(\begin{array}{cccc}
\frac{e_{b}^{1}(t)}{e_{b}^{1}(s)} & & & \\
& \frac{e_{b}^{2}(t)}{e_{b}^{2}(s)} & & \\
& & \ddots & \\
& & & \frac{e_{b}^{n}(t)}{e_{b}^{n}(s)}
\end{array}\right)
$$

Since only the amplitude of emission rates is considered as the optimization parameters, we establish the dynamic model of emission rates subject to the above background evolution

$$
\delta e(t)=M_{e}(t, s) \delta e(s), \quad s \leqslant t
$$

Several studies (e.g.[21]) stated that the estimation of the variable $x$ by the fix-interval Kalman smoother generally equals to the conditional expectation based on the observations within the entire time interval, denoted by $\mathbf{E}\left[x \mid\left\{y\left(t_{o b s}\right), t_{o b s} \in\left[t_{0}, t_{N}\right]\right\}\right]$. With the emission model (8), the estimate of $e(t)$ by Kalman smoother on $\left[t_{0}, t_{N}\right]$ follows the linear property of the conditional expectation,

$$
\begin{aligned}
& \mathbf{E}\left[e(t) \mid\left\{y\left(t_{o b s}\right), t_{o b s} \in\left[t_{0}, t_{N}\right]\right\}\right] \\
= & \mathbf{E}\left[M_{e}(t, s) e(s) \mid\left\{y\left(t_{o b s}\right), t_{o b s} \in\left[t_{0}, t_{N}\right]\right\}\right] \\
= & M_{e}(t, s) \mathbf{E}\left[e(s) \mid\left\{y\left(t_{o b s}\right), t_{o b s} \in\left[t_{0}, t_{N}\right]\right\}\right] .
\end{aligned}
$$

It implies that BLUEs of emission rates with the dynamic model (8) preserve the same diurnal profiles of the background of emission rates.

By rewriting (3) as $\delta c(t)=M\left(t, t_{0}\right) \delta c\left(t_{0}\right)+\int_{t_{0}}^{t} M(t, s) M_{e}\left(s, t_{0}\right) \delta e\left(t_{0}\right) d s$, we obtain the transport model with the state vector extended by emission rates

$$
\left(\begin{array}{l}
\delta c(t) \\
\delta e(t)
\end{array}\right)=\left(\begin{array}{cc}
M\left(t, t_{0}\right) & \int_{t_{0}}^{t} M(t, s) M_{e}\left(s, t_{0}\right) d s \\
0 & M_{e}\left(t, t_{0}\right)
\end{array}\right)\left(\begin{array}{l}
\delta c\left(t_{0}\right) \\
\delta e\left(t_{0}\right)
\end{array}\right) .
$$


Typically, there is no direct observation for emissions, apart from the flux tower observations used for carbon dioxide, which are not considered here. Therefore, we can reformulate the observation mapping as

$$
\delta y(t)=\left(H(t), 0_{n \times n}\right)\left(\begin{array}{c}
\delta c(t) \\
\delta e(t)
\end{array}\right)+\nu(t),
$$

where $0_{n \times n}$ is a $n \times n$ matrix with zero elements.

It is now clear that both concentrations and emission rates are included in the state vector of the homogeneous model (10), It allows us to apply the Kalman smoother in a fixed time interval $\left[t_{0}, t_{N}\right]$ in order to optimize both parameters. Besides, a more general case of the transport model extended by emission is shown in Appendix A.

\section{The degree of freedom for signal of concentrations and emissions}

In this section we will introduce the theoretical approach to determine the DFS of concentrations and emissions, resting on the extended model in Section 2. This approach gives us access to determine the potential ability of observations to optimize each variable of the above extended model, based on the Kalman smoother within a finite-time interval.

For convenience, we generalize the atmospheric transport model (10) by the following discrete-time linear system on the time interval $\left[t_{0}, t_{1}, \cdots, t_{N}\right]$ :

$$
\begin{aligned}
& x\left(t_{k+1}\right)=M\left(t_{k+1}, t_{k}\right) x\left(t_{k}\right)+\varepsilon\left(t_{k}\right), \\
& y\left(t_{k}\right)=H\left(t_{k}\right) x\left(t_{k}\right)+\nu\left(t_{k}\right),
\end{aligned}
$$

where $x(\cdot) \in \mathbf{R}^{n}$ is the state variable and $y\left(t_{k}\right) \in \mathbf{R}^{m\left(t_{k}\right)}$ is the observation vector at time $t_{k}$. The model error $\varepsilon\left(t_{k}\right)$ and the observation error $\nu\left(t_{k}\right)$, $k=1, \cdots, N$ of Gaussian distributions have zero means. The model error covariance matrix is denoted by $Q\left(t_{k}\right)$, while the observation error covariance matrix is denoted by $R\left(t_{k}\right)$.

According to Appendix B, applying the singular value decomposition into $P^{\frac{1}{2}}\left(t_{0} \mid t_{-1}\right) \mathcal{G}^{\top} \mathcal{R}^{-\frac{1}{2}}=V S U^{\top}$, we obtain

$$
\tilde{P}=P^{-\frac{1}{2}}\left(t_{0} \mid t_{-1}\right)\left(P\left(t_{0} \mid t_{-1}\right)-P\left(t_{0} \mid t_{N}\right)\right) P^{-\frac{1}{2}}\left(t_{0} \mid t_{-1}\right)=\sum_{i=1}^{r} \frac{s_{i}^{2}}{1+s_{i}^{2}} v_{i} v_{i}^{\top}
$$

where $v_{i}$ is the $i^{t h}$ left singular vector in $V$ related to the singular value $s_{i}$, which is the $i^{t h}$ element on the diagonal of $S$.

It is clear that the trace of $\tilde{P}$ can be used to evaluate the total improvements of model states. Thus, the nuclear norm is appropriately taken as the metric, which is defined as

$$
\|A\|_{1}=\operatorname{tr}\left(\sqrt{A^{\top} A}\right),
$$

where $A$ is any matrix and $\operatorname{tr}(\cdot)$ denotes the trace of the matrix. 
From (14), we obtain

$$
\|\tilde{P}\|_{1}=\operatorname{tr}(\tilde{P})=\sum_{i=1}^{r} \frac{s_{i}^{2}}{1+s_{i}^{2}}
$$

This is well-known as the degree of freedom for signal (DFS) of the model (e.g [38]).

It is obvious that $\|\tilde{P}\|_{1}<\|I\|_{1}=n$. Here $n$ can be considered as the total relative improvement if the system is definitely observed. Thus, if we consider the ratio

$$
\tilde{p}=\frac{\|\tilde{P}\|_{1}}{\|I\|_{1}}=\frac{\|\tilde{P}\|_{1}}{n} \in[0,1),
$$

the percentage of the total improvement of the model is obtained, which is henceforth called the relative degree of freedom for signal.

In order to get a deeper insight into the potential capacity of the observation network to improve the estimation of all model states, we consider the corresponding value in the diagonal of $\tilde{P}$ as the contribution of the degree of freedom for signal. Denote the $j^{\text {th }}$ element on the diagonal of $\tilde{P}$ by $\tilde{P}_{j}$, from (47), the contribution of the $j^{\text {th }}$ element of $x\left(t_{0}\right)$ to the degree of freedom for signal can be expressed as

$$
\tilde{P}_{j}=\sum_{i=1}^{r} \frac{s_{i}^{2}}{1+s_{i}^{2}}\left(v_{i j}\right)^{2}
$$

where $v_{i j}$ is the $j^{\text {th }}$ element of $v_{i}$.

Besides, we can see that Eqn. (14) enables us to discriminate the DFS contributed to different optimization parameters, which are here emission rates and initial values. Without loss of generality, we divide (14) into the following block matrix according to the dimension of $c$ and $e$

$$
\tilde{P}=\left(\begin{array}{cc}
\tilde{P}^{c} & \tilde{P}^{c e} \\
\tilde{P}^{e c} & \tilde{P}^{e}
\end{array}\right)=\sum_{i=1}^{2 n} \frac{s_{i}^{2}}{1+s_{i}^{2}}\left(\begin{array}{c}
v_{i}^{c} \\
v_{i}^{e}
\end{array}\right)\left(v_{i}^{c^{\top}}, v_{i}^{e^{\top}}\right) \in \mathbf{R}^{2 n \times 2 n},
$$

where $\left(v_{i}^{c^{\top}}, v_{i}^{e^{\top}}\right)^{\top}=v_{i}$.

It is easy to see that

$$
\tilde{P}^{c}=\sum_{i=1}^{2 n} \frac{s_{i}^{2}}{1+s_{i}^{2}} v_{i}^{c} v_{i}^{c^{\top}}, \quad \tilde{P}^{e}=\sum_{i=1}^{2 n} \frac{s_{i}^{2}}{1+s_{i}^{2}} v_{i}^{e} v_{i}^{e^{\top}} .
$$

Further, the degree of freedom for signal of $j^{t h}$ element in $c\left(t_{0}\right)$ and $e\left(t_{0}\right)$ are given by

$$
\tilde{P}_{j}^{c}=\sum_{i=1}^{2 n} \frac{s_{i}^{2}}{1+s_{i}^{2}}\left(v_{i j}^{c}\right)^{2}, \quad \tilde{P}_{j}^{e}=\sum_{i=1}^{2 n} \frac{s_{i}^{2}}{1+s_{i}^{2}}\left(v_{i j}^{e}\right)^{2},
$$

where $v_{i j}^{c}$ and $v_{i j}^{e}$ are the $j^{t h}$ elements of $v_{i}^{c}$ and $v_{i}^{e}$, respectively. 
Moreover, the degree of freedom for signal of concentration $\left\|\tilde{P}^{c}\right\|_{1}$ and emission rates $\left\|\tilde{P}^{e}\right\|_{1}$ are calculated by

$$
\left\|\tilde{P}^{c}\right\|_{1}=\sum_{i=1}^{2 n} \frac{s_{i}^{2}}{1+s_{i}^{2}} \operatorname{tr}\left(v_{i}^{c} v_{i}^{c^{\top}}\right), \quad\left\|\tilde{P}^{e}\right\|_{1}=\sum_{i=1}^{2 n} \frac{s_{i}^{2}}{1+s_{i}^{2}} \operatorname{tr}\left(v_{i}^{e} v_{i}^{e^{\top}}\right) .
$$

It is worth noticing that

$$
\begin{aligned}
& \tilde{P}^{c}=\left(P^{c}\left(t_{0} \mid t_{-1}\right)\right)^{-\frac{1}{2}}\left(P^{c}\left(t_{0} \mid t_{-1}\right)-P^{c}\left(t_{0} \mid t_{N}\right)\right)\left(P^{c}\left(t_{0} \mid t_{-1}\right)\right)^{-\frac{1}{2}} \\
& \tilde{P}^{e}=\left(P^{e}\left(t_{0} \mid t_{-1}\right)\right)^{-\frac{1}{2}}\left(P^{e}\left(t_{0} \mid t_{-1}\right)-P^{e}\left(t_{0} \mid t_{N}\right)\right)\left(P^{e}\left(t_{0} \mid t_{-1}\right)\right)^{-\frac{1}{2}}
\end{aligned}
$$

if and only if there is no prior correlation between the initial concentration and emission rates. In this case $P^{c e}\left(t_{0} \mid t_{-1}\right)=0_{n \times n}$, the corresponding relative degrees of freedom for signal of concentration and emission rates are defined as

$$
\tilde{p}^{c}=\frac{\left\|\tilde{P}^{c}\right\|_{1}}{n}, \quad \tilde{p}^{e}=\frac{\left\|\tilde{P}^{e}\right\|_{1}}{n} .
$$

From (17), $\tilde{p}^{c} \in[0,1)$ and $\tilde{p}^{e} \in[0,1)$ seem like percentages of the relative improvements of concentration and emission rates, respectively. However, efficient observation networks ideally lead to values are close to 1 for both of them, such that

$$
\frac{\left\|\tilde{P}^{c}\right\|_{1}}{n}+\frac{\left\|\tilde{P}^{e}\right\|_{1}}{n}>1 .
$$

It results from the reason that the normalization of $\tilde{P}$ is only with respect to the extended covariance matrix $P\left(t_{0} \mid t_{-1}\right)$ rather than specified to the covariance matrices $\tilde{P}^{c}\left(t_{0} \mid t_{-1}\right)$ and $\tilde{P}^{e}\left(t_{0} \mid t_{-1}\right)$. The relative degree of freedom for signal cannot serve our objective to distinguish the observability of concentration and emission rates. By observing the block form of $\tilde{P}$, we have

$$
\left\|\tilde{P}^{c}\right\|_{1}+\left\|\tilde{P}^{e}\right\|_{1}=\|\tilde{P}\|_{1} .
$$

Thus, in order to compare the potential improvements of the concentration and emission rates separately, we define a relative ratio of the degree of freedom for signal for concentrations or emission rates as

$$
\tilde{p}^{c}=\frac{\left\|\tilde{P}^{c}\right\|_{1}}{\|\tilde{P}\|_{1}}, \quad \tilde{p}^{e}=\frac{\left\|\tilde{P}^{e}\right\|_{1}}{\|\tilde{P}\|_{1}}, \quad \tilde{p}^{e}+\tilde{p}^{c} \equiv 1 .
$$

If the degree or relative degree of freedom for signal of the observation network and assimilation window is almost zero, an improvement cannot be expected. In contrast, $\left\{\tilde{P}_{j}^{c}\right\}_{j=1}^{n}$ and $\left\{\tilde{P}_{j}^{e}\right\}_{j=1}^{n}$, which show the improvement of each parameter $j$ of concentrations and emission rates respectively, can help us determining which parameters can be expected to be optimized by the existing observation configurations. Furthermore, comparing $\tilde{p}^{c}$ with $\tilde{p}^{e}$, we can conclude that the estimate of the one with the larger relative ratio of freedom for signal can be improved more efficiently by the existing observation 
configurations than the other. In other words, if $\tilde{p}^{c}>\tilde{p}^{e}$, the existing observation configuration is more sensitive to the initial values of concentrations. Conversely, if $\tilde{p}^{c}<\tilde{p}^{e}$, the observation configurations can improve the estimate of emission rates better. According to $\tilde{p}^{c}$ and $\tilde{p}^{e}$, the relative weights between the concentrations and emission rates can be identified quantitatively. In a data assimilation context, where observations are in a weighted relation to the background, the BLUE favors those parameters with higher observation efficiency.

The special case with $\tilde{p}^{e}$ being very close to zero implies that observation network is not detectable for emission rate optimization.

\section{The ensemble approach to determine the DFS}

The ensemble Kalman smoother (EnKS), as a Monte Carlo implementation derived from the Kalman smoother, is a frequently applied tool for problems with a large number of control variables in the field of data assimilation [1], [18]. In this section, in order to identify the potential capacities of observation networks to optimize the concentration and especially the poorly known emission rates for high-dimensional problems, we will introduce the ensemble-based version of the approach in Section 3.

According to Appendix C, analog to Section 3, we have

$$
\bar{P}=\bar{P}^{\dagger \frac{1}{2}}\left(t_{0} \mid t_{-1}\right)\left(\bar{P}\left(t_{0} \mid t_{-1}\right)-\bar{P}\left(t_{0} \mid t_{N}\right)\right) \bar{P}^{\dagger \frac{1}{2}}\left(t_{0} \mid t_{-1}\right)=\sum_{i=1}^{r} \frac{\bar{s}_{i}^{2}}{1+\bar{s}_{i}^{2}} \bar{v}_{i} \bar{v}_{i}^{\top}
$$

Similar to Section 3, we can also divide $\bar{P}$ into the block form according to the dimensions of the concentration and emission rates. Thus, we correspondingly obtain the ensemble degree of freedom for signal of $j^{\text {th }}$ element in $c\left(t_{0}\right)$ and $e\left(t_{0}\right)$

$$
\bar{P}_{j}^{c}=\sum_{i=1}^{r} \frac{\bar{s}_{i}^{2}}{1+\bar{s}_{i}^{2}}\left(\bar{v}_{i j}^{c}\right)^{2}, \quad \bar{P}_{j}^{e}=\sum_{i=1}^{r} \frac{\bar{s}_{i}^{2}}{1+\bar{s}_{i}^{2}}\left(\bar{v}_{i j}^{e}\right)^{2},
$$

where $\bar{v}_{i j}^{c}$ and $\bar{v}_{i j}^{e}$ are the $j^{\text {th }}$ elements of $\bar{v}_{i}^{c}$ and $\bar{v}_{i}^{e}$, respectively and

$$
\left(\bar{v}_{i}^{c^{\top}}, \bar{v}_{i}^{e^{\top}}\right)^{\top}=\bar{v}_{i}
$$

We observe that (29) and (14) have a similar form. By virtue of

$$
\bar{P}^{\dagger \frac{1}{2}}\left(t_{0} \mid t_{-1}\right) \bar{P}_{x y}^{f} \overline{\mathcal{R}}^{-\frac{1}{2}}=\bar{P}^{\frac{1}{2}}\left(t_{0} \mid t_{-1}\right) \mathcal{G}^{\top} \overline{\mathcal{R}}^{-\frac{1}{2}}
$$

we can find that the final results of (14) and (63) are equivalent. However, compared with $P^{\frac{1}{2}}\left(t_{0} \mid t_{-1}\right) \mathcal{G}^{\top} \mathcal{R}^{-\frac{1}{2}}$, the ensemble expression $\bar{P}^{\dagger \frac{1}{2}}\left(t_{0} \mid t_{-1}\right) \bar{P}_{x y}^{f} \overline{\mathcal{R}}^{-\frac{1}{2}}$ processes the absolute advantage that in the calculation of $\bar{P}_{x y}^{f}$ since we do not need the explicit form of $\mathcal{G}$. It allows us to code it line by line such that our approach is computationally more efficient. 
Analog to the standard formulation, we can similarly define the ensemble degree of freedom for signal (EnDFS) as $\|\bar{P}\|_{1}$ and consider each element on the diagonal of $\bar{P}$ as the contribution to EnDFS of the corresponding model state.

Since $\bar{P}\left(t_{0} \mid t_{-1}\right)$ does not typically have the full rank,

$$
\begin{aligned}
& \bar{P}^{\dagger \frac{1}{2}}\left(t_{0} \mid t_{-1}\right)\left(\bar{P}\left(t_{0} \mid t_{-1}\right)-\bar{P}\left(t_{0} \mid t_{N}\right)\right) \bar{P}^{\dagger \frac{1}{2}}\left(t_{0} \mid t_{-1}\right) \\
= & V_{0} \hat{S}_{0}^{\dagger} V_{0}^{\top}\left(V_{0} \hat{S}_{0}^{2} V_{0}^{\top}\right) V_{0} \hat{S}_{0}^{\dagger} V_{0}^{\top}-\bar{P}^{\dagger \frac{1}{2}}\left(t_{0} \mid t_{-1}\right) \bar{P}\left(t_{0} \mid t_{N}\right) \bar{P}^{\dagger \frac{1}{2}}\left(t_{0} \mid t_{-1}\right) \\
= & V_{0} I_{r_{0}} V_{0}^{\top}-\bar{P}^{\dagger \frac{1}{2}}\left(t_{0} \mid t_{-1}\right) \bar{P}\left(t_{0} \mid t_{N}\right) \bar{P}^{\dagger \frac{1}{2}}\left(t_{0} \mid t_{-1}\right),
\end{aligned}
$$

where $I_{r_{0}}$ is the diagonal matrix with the diagonal $\left(\mathbf{1}_{1 \times r_{0}}, 0_{1 \times\left(n-r_{0}\right)}\right)$. It is clear from $(60)$ that $\bar{P}^{\dagger \frac{1}{2}}\left(t_{0} \mid t_{-1}\right) \bar{P}\left(t_{0} \mid t_{N}\right) \bar{P}^{\dagger \frac{1}{2}}\left(t_{0} \mid t_{-1}\right)$ is still a nonnegative definite matrix.

Thus, the ensemble relative degree of freedom for signal (EnRDFS) is defined through

$$
\bar{p}=\frac{\|\bar{P}\|_{1}}{\left\|I_{r_{0}}\right\|_{1}}=\frac{\|\bar{P}\|_{1}}{r_{0}} \in[0,1) .
$$

In order to distinguish the improvements for concentrations and emission rates, the ensemble relative ratios of DFS remain

$$
\bar{p}^{c}=\frac{\left\|\bar{P}^{c}\right\|_{1}}{\|\bar{P}\|_{1}}, \quad \bar{p}^{e}=\frac{\left\|\bar{P}^{e}\right\|_{1}}{\|\bar{P}\|_{1}} .
$$

\section{Sensitivity of observation networks}

The above discussion about DFS aims to evaluate a predefined measurement network on its potential to analyze initial values and emission rates simultaneously. In Appendix D, independent of any concrete data assimilation method, we use the singular vector approach (see [8], [9],[31] etc.) to identify the sensitive directions of observation networks to initial values and emission rates separately and show the association with Section 3.

From Appendix D, we can see that the singular value $s_{k}$ shows the amplification of the impact of the initial state to the observation configurations during the entire time interval. The associated singular vector in the state space $v_{k}$ is the direction of the $\mathrm{k}^{\text {th }}$ growth of the perturbation of observations, which evolved from the initial perturbation. With the special choice $W_{0}=P^{-1}\left(t_{0} \mid t_{-1}\right)$ and $\mathcal{W}=\mathcal{R}^{-1}$, we compare the sensitivity analysis with the analysis in Section 3. Since the sequence $\left\{\frac{s_{k}^{2}}{1+s_{k}^{2}}\right\}$ decreases with the decrease of $s_{k}, k=1, \cdots, n$, it is clear that the vector $v_{k}$ also points to the $\mathrm{k}^{\text {th }}$ direction which maximizes the relative improvement of estimates based on the Kalman smoother. It indicates that the states with higher contributions to DFS are the same with the states, which are more sensitive to the observation 
networks. Besides, the leading singular value $s_{1}$ is related to the operator norm of $\tilde{P}$ as

$$
\|\tilde{P}\|=\max _{\|x\|=1}\|\tilde{P} x\|=\frac{s_{1}^{2}}{1+s_{1}^{2}}
$$

which implies the upper boundedness of $\tilde{P}$. It gives us an access to approximate and target the sensitive parameters or areas with the metric of the leading singular vectors weighted by the corresponding singular values.

Moreover, due to the homogeneity of the atmospheric transport model state vector extended with emissions, the above sensitivity analysis can be easily applied by dividing singular vectors into the block form according to the dimensions of the initial state and emissions. The corresponding block parts of different singular vectors indicate the different sensitive directions of the initial state and emissions and allow for this relative quantification. Correspondingly, we can approximate and target the parameters sensitive to the existing observation networks for both initial values and emission rates.

\section{Example}

In this section, we apply the approaches in Section 4 and Section 5 in to an elementary advection-diffusion model to show how to assess the potential observability of concentrations and emission rates through EnDFS. We can see how it helps to identify the sensitive elements of both concentrations and emission rates to the given observations. We consider a linear advection-diffusion model with Dirichlet horizontal (lateral) boundary condition and Neumann lower (surface) boundary condition in the vertical direction on the domain $[0,14] \times[0,14] \times[0,4]$, reading

$$
\frac{\partial \delta c}{\partial t}=-v_{x} \frac{\partial \delta c}{\partial x}-v_{y} \frac{\partial \delta c}{\partial y}+\frac{\partial}{\partial z}\left(K(z) \frac{\partial \delta c}{\partial z}\right)+\delta e,
$$

where $\delta c, \delta e$ are the perturbations of the concentration, and the emission rates of passive tracer respectively. For vertical diffusion, $K(z)$ is a differentiable function of height $z$.

For velocity $v_{x}=v_{y}=0.5$ and the time step $\Delta t=0.5$, the numerical solution is based on the symmetric operator splitting technique [48] with the following operator sequence

$$
\delta c(t+\triangle t)=T_{x} T_{y} D_{z} A D_{z} T_{y} T_{x} \delta c(t)
$$

where $T_{x}$ and $T_{y}$ are transport operators in horizontal directions $x$ and $y, D_{z}$ is the diffusion operator in vertical direction $z$. The parameters of emission and deposition rates are included in $A$. The Lax-Wendroff algorithm is chosen as the discretization method for horizontal advection with $\triangle x=\triangle y=1$. The vertical diffusion is discretized with $\triangle z=1$ by Crank-Nicolson scheme with the Thomas algorithm [24] as solver. The number of the grid points is $N_{g}=1125$. 
With the same temporal and spatial discretization of the concentration, the background knowledge of the emission rate is given by $e_{b}\left(t_{n}, i, j, l\right)$, where $n=1, \cdots, N$. We establish the discrete dynamic model of the emission rate according to $(8)$

$$
\delta e\left(t_{n+1}\right)=M_{e}\left(t_{n+1}, t_{n}\right) \delta e\left(t_{n}\right), \quad n=1, \cdots, N,
$$

where $M_{e}\left(t_{n+1}, t_{n}\right)=e_{b}\left(t_{n+1}\right) / e_{b}\left(t_{n}\right)$.

For expository reasons we assume $\delta d$ is a constant over time and the observation operator $H(t)$ mapping the state space to the observation space is a $1 \times 2 N_{g}$ time-invariant matrix. In the paper by [46], the convergence of the numerical solution based on the above splitting and dicretization method to the original solution of the partial differential equation (36) has been proved.

In our simulations, we produce $q=500$ (the ensemble number) samples for the initial concentration and emission rate respectively, by pseudo independent random numbers and make the states correlated by the moving average technique. It has been tested that the computation cost of our approach is linearly increasing with the number of ensembles. In the following, we present three different tests, aiming to demonstrate the roles of variable winds, emissions, and vertical diffusion.

Advection tests: The following part demonstrates the potential capacity and limits of the DFS analysis tool. The prototypical examples are designed to show the expected elementary outcomes of the following situations, which exhibit the effects of assimilation window length in relation to emission location: these include (i) an assimilation window is too short to capture emission impacts at the observation site, (ii) an extended assimilation window with balanced signal of impacts of concentrations and emissions at the observation site, and (iii) a further increased assimilation window featuring a declining impact of initial values and growing emission impact. The first elementary advection test (Fig. 1 to Fig. 7) identifies the sensitivities of parameters subject to different wind direction and data assimilation window (DAW) through the EnDFS of each element of the concentrations and emission rates. Focusing on the advection effects, we apply the model with a weak diffusion process $\left(K(z)=0.5 e^{-z^{2}}\right)$.

In Fig. 1 to 3 we assume southwesterly winds and the assigned data assimilation windows are $10 \triangle t, 35 \triangle t$, and $48 \triangle t$, respectively. The computation times are approximately $8.1 \mathrm{~s}, 28.5 \mathrm{~s}$ and $39.4 \mathrm{~s}$ in our tests with the above three different assimilation windows, from which we can verify that the computation cost is nearly linearly increasing with the length of data assimilation window. The contributions to EnDFS of the initial states are shown in the left panels of Fig. 1 to 3 . We can find that in the horizontal fields at lowest layer $(z=0)$, the optimized field of the concentration is enlarged with the extension of data assimilation windows. This is because an increased domain of the concentration are controlled with longer data assimilation windows.

The right panels of Fig. 1 to 3 show the ensemble degree of freedom for signal (EnDFS) of the emission rate at each grid point with $z=0$. From the right panel of Fig. 1, we can observe that the contributions to EnDFS from 
emissions are less than $2 \times 10^{-3}$. Compared with the right panel of Fig. 1, it is obvious that the EnDFS of the emissions are smaller than the EnDFS of the concentration in the influenced area. It indicates that the observations cannot detect the emission rate within $10 \triangle t$ data assimilation window. Thus, in this case initial values of the area adjacent to the observation site are alone optimized. It is shown in the right panels of Fig. 2 and Fig. 3 that the emission rates plays a more and more important role on the impact of observations. In this two cases, we consider both the concentration and emission rate as optimizable parameters. The quantitative balance between the concentration and emission rate is provided in Table 1.

In its upper row panels, Fig. 4 exhibits the singular values underlying the results shown in Fig. 1 to 3. We approximate the sensitivities of the initial concentrations by the first five leading singular vectors weighted by the associated singular values in the nuclear norm and show the results in the three panels in the lower row of Fig. 4. It is clear that the sensitive area can be well targeted by only few singular vectors, although the sensitivity analysis cannot provide the quantitative solutions with a clear statistical significance as the the degree of freedom for signal of the model. Besides, it corresponds to expectations that the areas of influence to the measurement site depends on wind direction and assimilation window lengths, which corresponds to expectations.

As the counter examples, Fig. 5 to 7 also show the EnDFS of the concentration and emission rate under the same assumptions as Fig. 1 to 3 respectively, except that northeasterly wind is assumed. Clearly, with this adverse wind direction, whatever the duration of the assimilation window is, the emission is not detectable and improvable by that particular observation configuration. This hypothesis is demonstrated by our method. The quantitative balances of the related figures are exposed in Table 1 It can be seen that the insensitivity to emission rate optimisation remains equally low and affected by numerical noise. .

Emission signal tests: The purpose of emission signal tests (Fig. 8 and Fig. 9) is to assess the impact of observation configurations to the emission rates evolved with different diurnal profiles. We make the same assumptions as for Fig. 3 except that the wind speed in Fig. 8 and Fig. 9 is accelerated such that the profiles of the emission rate is better detectable in relation to the observation within the assimilation window $48 \triangle t$. The only distinction between the situations in Fig. 8 and Fig. 9 is the pronounced diurnal cycle background profile of the emission rate during the assimilation window $48 \triangle t$. Since the profiles of emission rates are correlated with the emitted amount of that species during the data assimilation window, it is clearly shown in Table 1 that the distinct variation of the emission rate during the data assimilation window acts to level $\bar{p}^{c}$ and $\bar{p}^{e}$, and thus helps to improve the estimates of source.

Diffusion tests: The vertical exchange of trace gases can be described by advection and diffusion, dependent on the nature of the process and the model grid resolution. In this study we confine our simulation to include the vertical diffusion only for the vertical coupling. The diffusion tests (Fig. 10 to Fig. 12) 

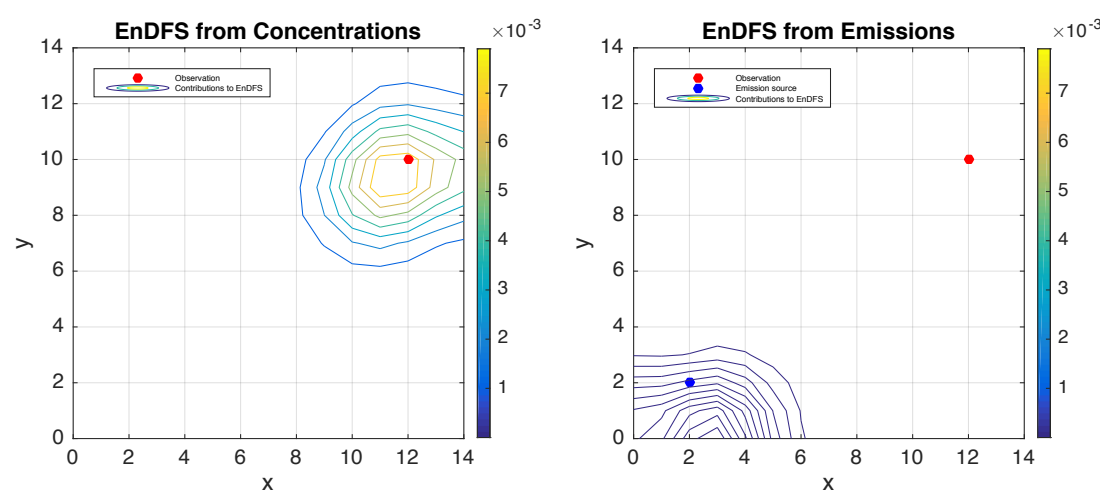

Fig. 1 Advection test with $10 \triangle t$ data assimilation window (DAW) and southwesterly wind. The contributions from the concentration and emission to EnDFS are shown in the left and right figure panels respectively. The blue point located at $(12,10,0)$ shows the time-invariant observation configuration. The blue point located at $(2,2,0)$ is the source of the emission rate.

aims to test our approach by comparing the EnDFS of the concentration and the emission rate at the layer $z=0$ with a weak diffusion process and a strong diffusion process. We assume that the observation configuration at each time step is located at $(12,10,4)$ in the diffusion test, with $K(z)=0.5 e^{-z^{2}}$ in Fig. 10 and $K(z)=0.5 e^{-z^{2}}+1$ in Fig. 11. Besides, Fig. 10 and Fig. 12 preserve the same assumptions with Fig. 3.

It is obvious from Fig. 3 and Fig. 10 that the different observation locations strongly influence the distribution of the concentration. Table 2 shows that with the same diffusion coefficient the ensemble degree of freedom for signal of the concentration in the lowest layer in Fig. 3 is definitely larger than the one in Fig. 10. Moreover, it can be seen from Table 1 that the observation configuration at the top layer is not efficient to the emission rate with such weak diffusion within $48 \triangle t$ data assimilation window .

Comparing Fig. 10 with Fig. 11, we can see how the EnDFS of concentration and emission rate increase with the stronger diffusion process. The increasing impact of the observation configuration with the stronger diffusion is also verified by the EnDFS and ensemble relative ratios of DFS of the concentration and emission rate for Fig. 10 and 11 in Table 2. The balances between the concentration and emission rate for Fig. 10 and 11 are shown in Table 1. The significant difference of the 'weight' of the emission rate in Table 1 implies that the observation configuration cannot detect the emission at the lowest layer with such a weak diffusion in Fig. 10, while with the stronger in Fig. 11 both the concentration and emission rate should be considered as optimized parameters with the corresponding 'weights'.

Finally, similar to Fig. 4, the singular values of Fig. 10, Fig. 11 and the approximating targeting results of sensitive parameters are shown in Fig. 12. It shows that the optimizable parameters can be also caught by few leading singular vectors in the diffusion tests. 

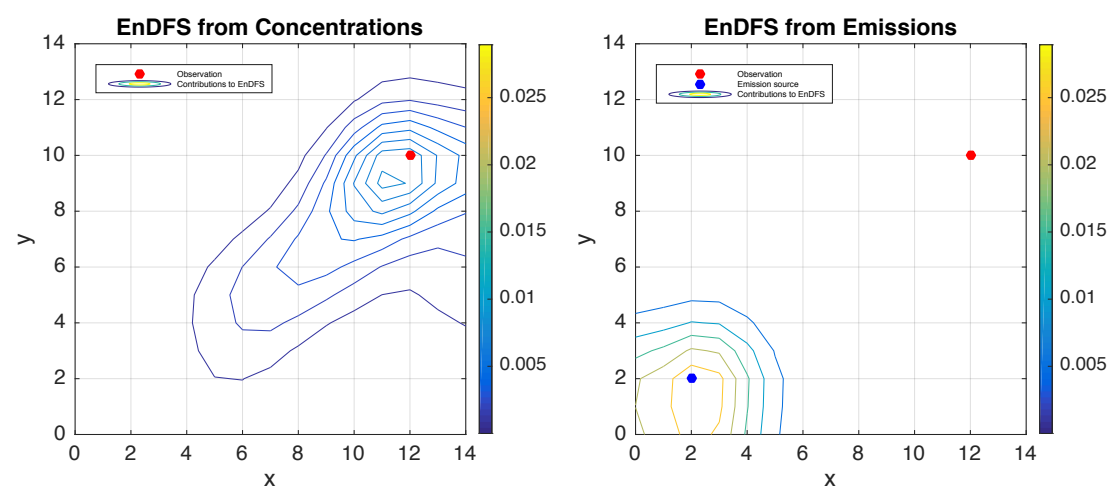

Fig. 2 Advection test with $35 \triangle t$ DAW and southwesterly wind. Plotting conventions are as in Fig. 1.
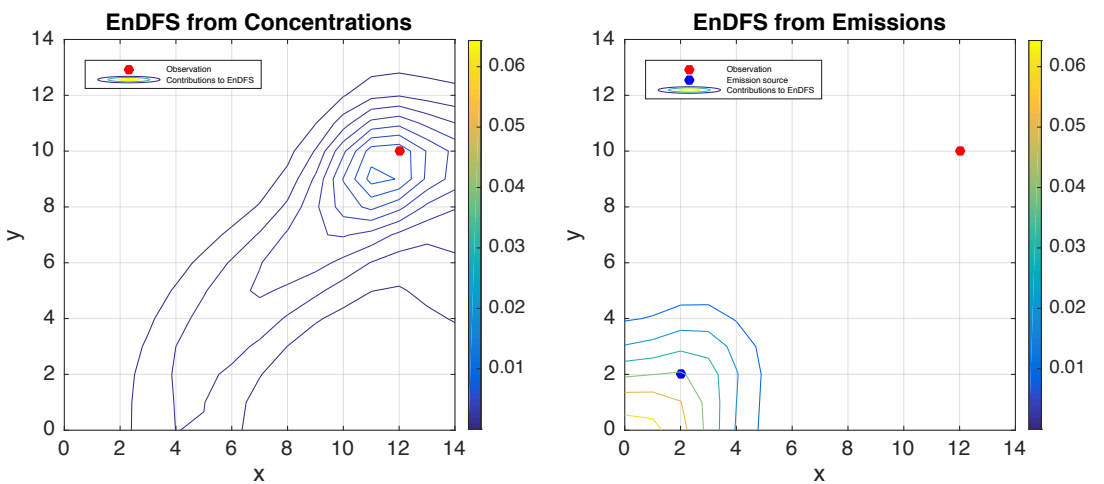

Fig. 3 Advection test with $48 \triangle t$ DAW and southwesterly wind. Plotting conventions are as in Fig. 1.

Table 1 Ensemble relative ratios of the initial value and emission rate at the lowest layer.

\begin{tabular}{c|cccccccccc}
\hline \hline & Fig. 1 & Fig. 2 & Fig. 3 & Fig. 5 & Fig. 6 & Fig. 7 & Fig. 8 & Fig. 9 & Fig. 10 & Fig. 11 \\
\hline $\bar{p}^{c}$ & 0.992 & 0.455 & 0.285 & 0.994 & 0.994 & 0.994 & 0.225 & 0.181 & 0.993 & 0.191 \\
$\bar{p}^{e}$ & 0.008 & 0.545 & 0.715 & 0.006 & 0.006 & 0.006 & 0.775 & 0.8192 & 0.007 & 0.890 \\
\hline
\end{tabular}

\section{Conclusions and Outlooks}

In this study we extended the transport-diffusion models forced by emission rates in a novel way. Based on a Kalman smoother technique, we developed an approach to the quantify the impact of a given observation network on the optimization of initial trace gas state and emission rates. The contribution to the degree of freedom for signal is adopted as a criterion to evaluate the potential improvement of each element in the extended state vector. The degree of freedom for signal and a number of metrics was taken as a quantitative solutions to measure to what extent the parameters can be optimized in advance of the 
Singular Values (10 $\Delta t$ DAW) Singular Values (35 $\Delta t$ DAW) Singular Values (48 $\Delta t$ DAW)
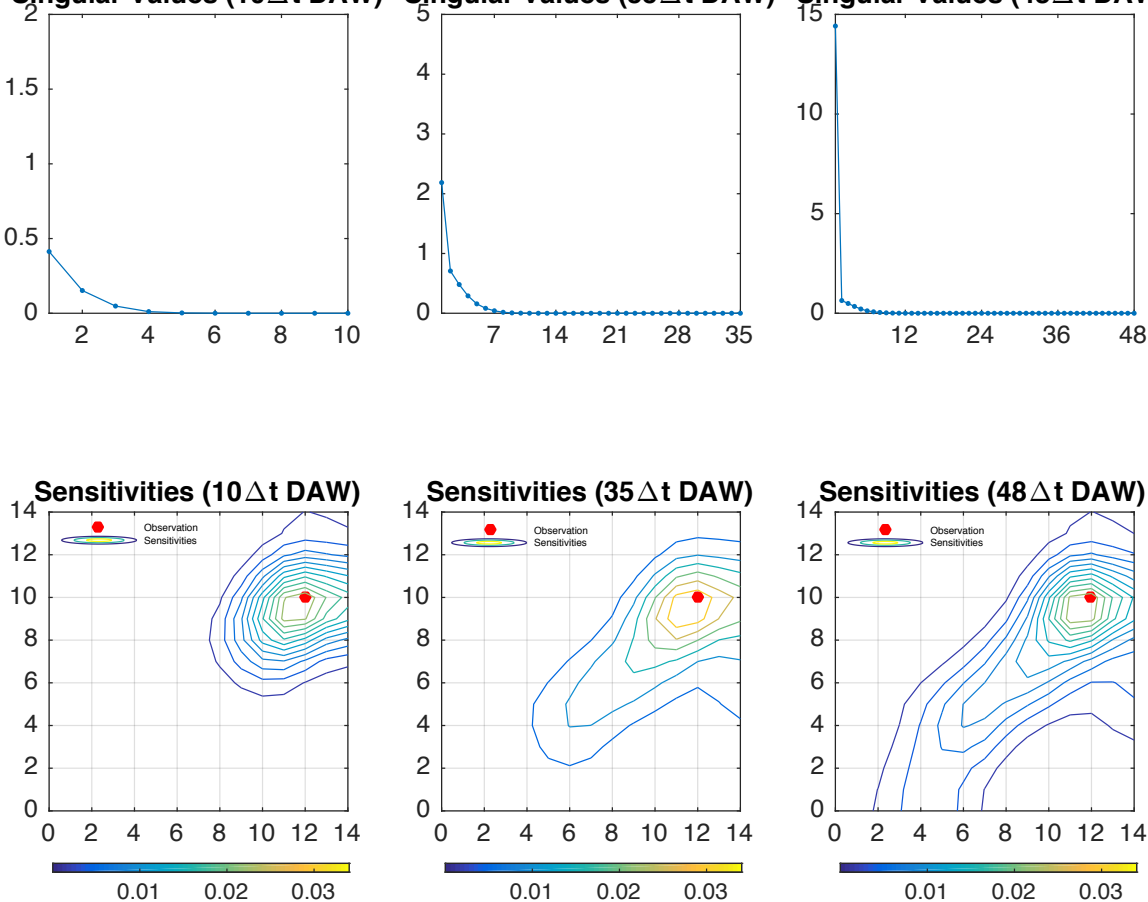

Fig. 4 The panels in the first row are singular values of the advenction tests shown in the left panels of Fig. $1 \sim 3$. The panels in the second row show the corresponding sensitivities of concentrations at the initial time approximated by 5 leading singular vectors.
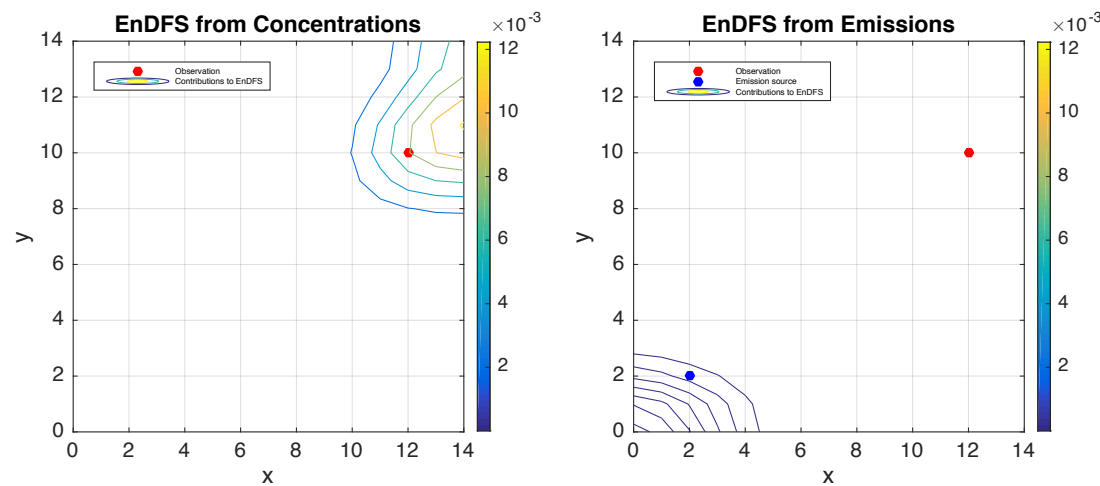

Fig. 5 Advection test with $10 \triangle t$ DAW and northeasterly wind. Plotting conventions are as in Fig. 1. 

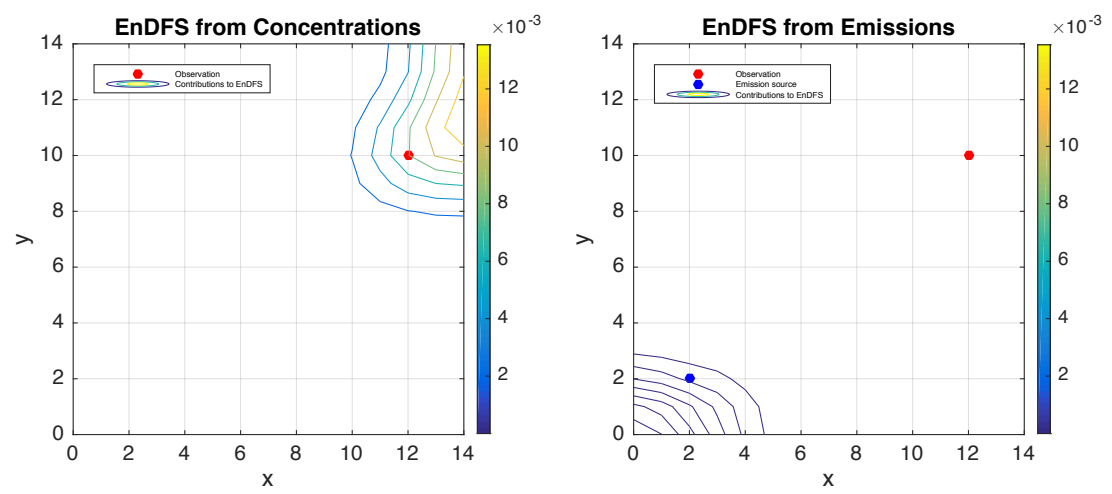

Fig. 6 Advection test with $35 \triangle t$ DAW and northeasterly wind. Plotting conventions are as in Fig. 1.
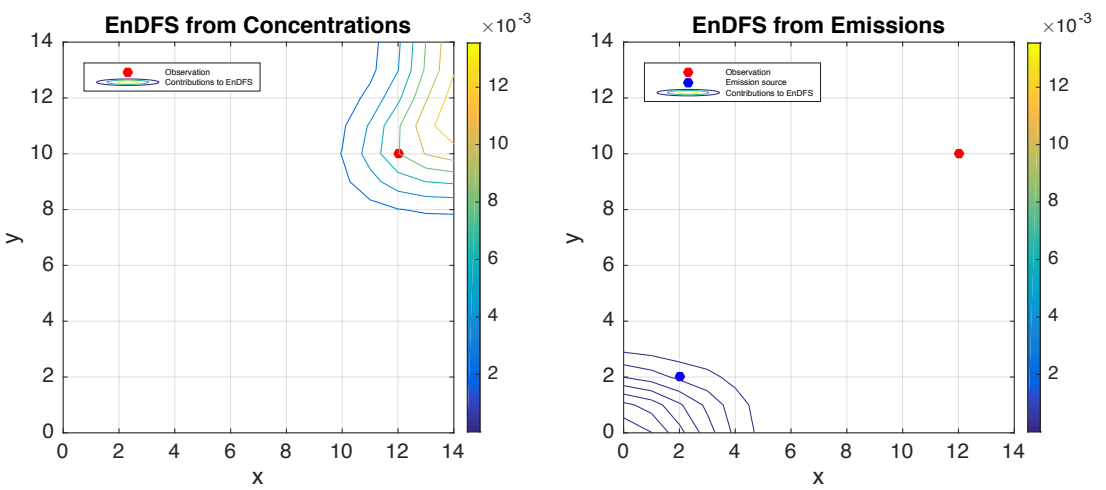

Fig. 7 Advection test with $48 \triangle t$ DAW and northeasterly wind. Plotting conventions are as in Fig. 1.
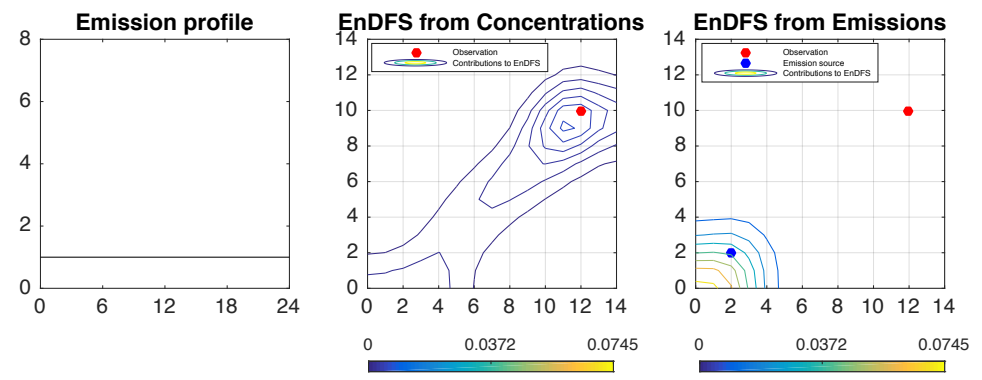

Fig. 8 Emission signal test (weak) with $48 \triangle t$ DAW and southwesterly wind ( $v_{x}=1$ and $\left.v_{y}=1\right)$. Plotting conventions are as in Fig. 1 . 

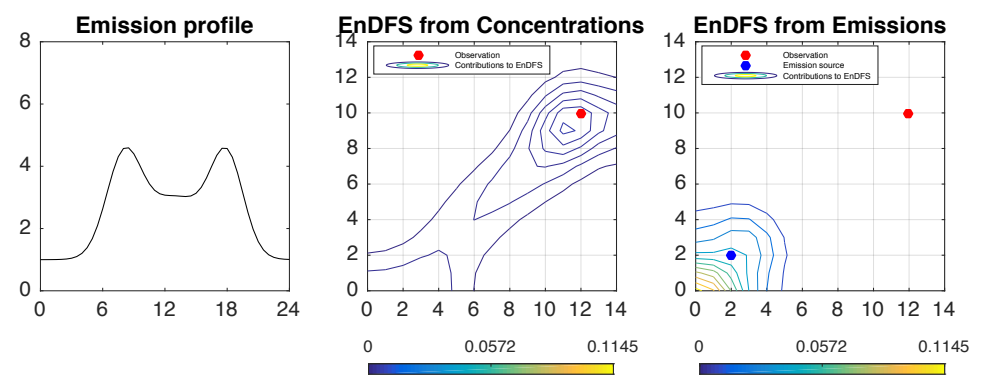

Fig. 9 Emission signal test (strong) with $48 \triangle t$ DAW and southwest wind $\left(v_{x}=1\right.$ and $\left.v_{y}=1\right)$. Plotting conventions are as in Fig. 1.
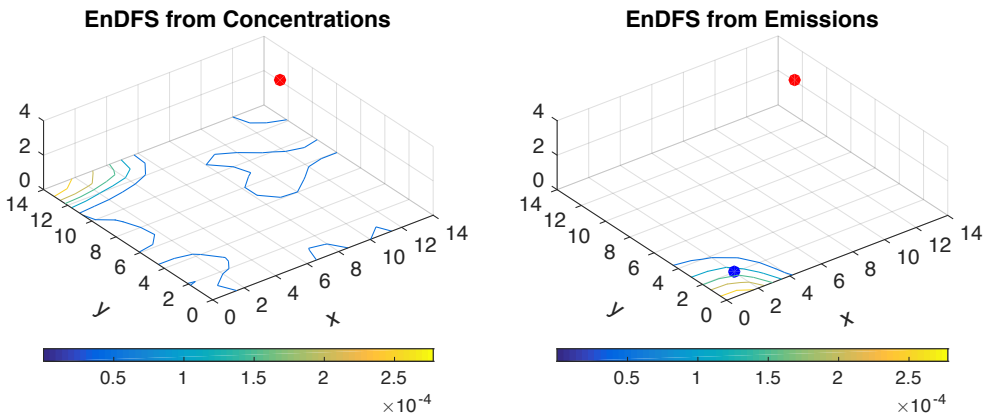

Fig. 10 Diffusion test (weak) with $48 \triangle t$ DAW and southwesterly wind. Plotting conventions are as in Fig. 1.
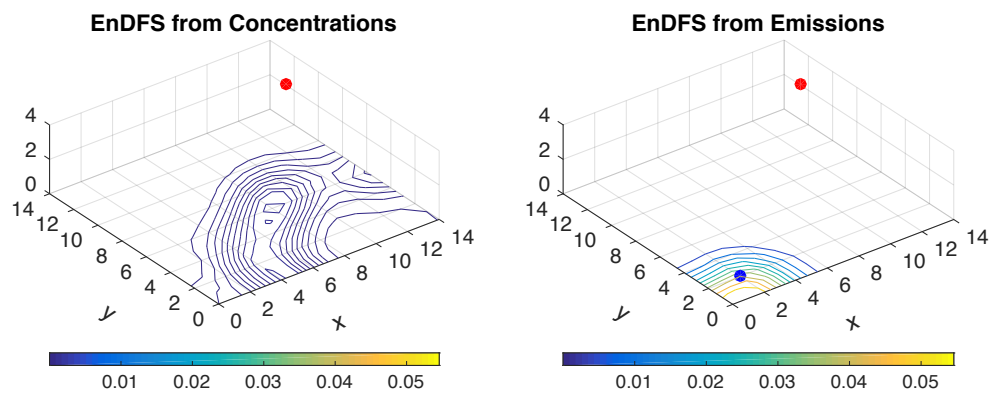

Fig. 11 Diffusion test (strong) with $48 \triangle t$ DAW and southwesterly wind. Plotting conventions are as in Fig. 1.

data assimilation procedure. The ensemble case of the approach gave us the feasibility to determine the assessment of the potential observability joint for initial values and emission rates for high-dimensional models. We formulated the sensitivity of observational networks by seeking the fastest directions of the perturbation ratio between initial states and observation configurations during the entire time interval. It facilitates to target the sensitive parameters to the observation networks by few leading singular values and vectors. A series of 

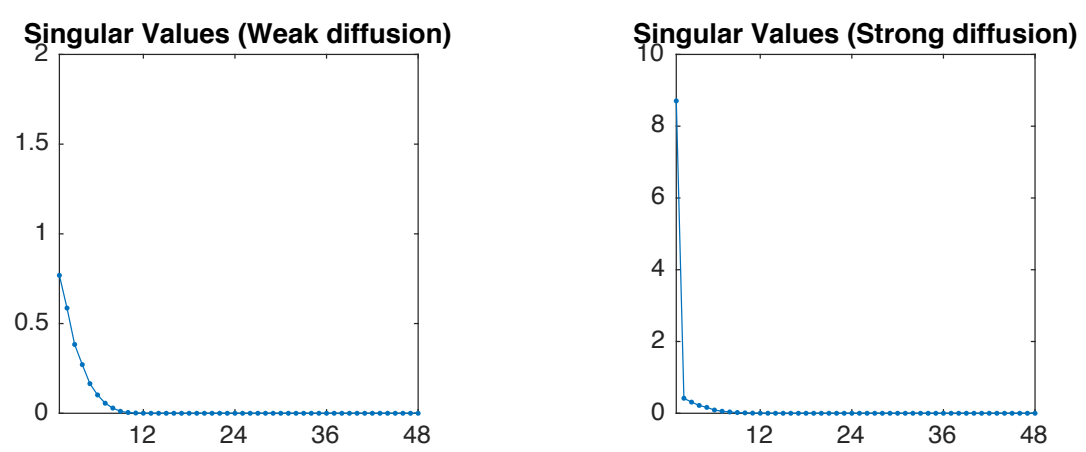

Sensitivities (Weak diffusion)

Sensitivities (Strong diffusion)
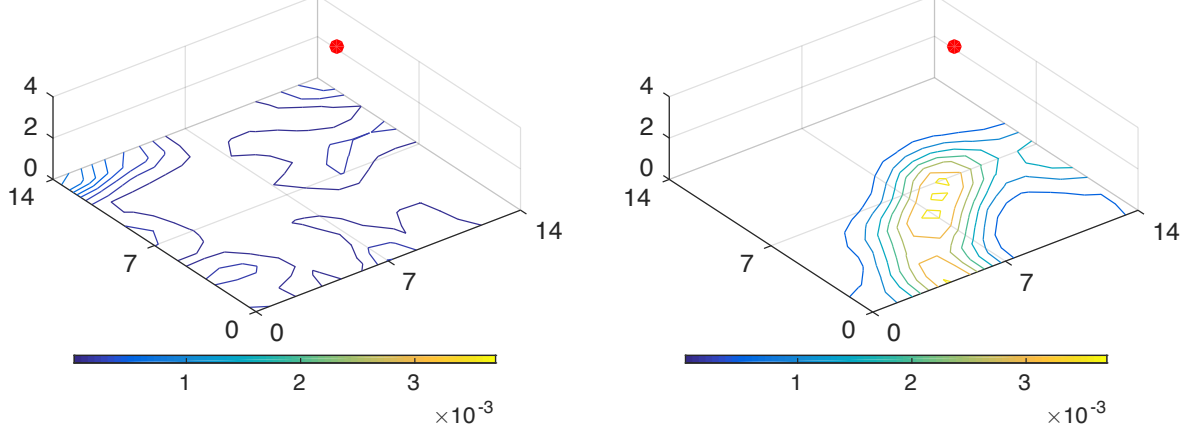

Fig. 12 The panels in the first row show singular values of the diffusion tests shown in the left panels of Fig. 10 and Fig. 11. The panels in the second row show sensitivities of concentrations at the initial time approximated by 5 leading singular vectors.

Table 2 The ensemble degrees of freedom for signal of the initial concentration and emission at the lowest layer.

\begin{tabular}{l|cc}
\hline \hline & $P_{\text {low }}^{c}$ & $P_{\text {low }}^{e}$ \\
\hline Fig. 3 & 0.277 & 0.885 \\
Fig. 10 & 0.010 & 0.003 \\
Fig. 11 & 0.050 & 0.789 \\
\hline \hline
\end{tabular}

experiments based on an elementary advection-diffusion model illustrated the significance of our approach in different situations.

In the future, we plan to apply this approach into the real atmospheric transport model to solve practical network validation problems prior to the solution of the inversion task, as far as the validity of the tangent linear assumption holds. 


\section{Appendix A}

In this appendix, we show a more general case to extend the control vector by emissions. As we know, the initial state and emission rates do not have the same dimension in some practical cases, as there can be more than one different kinds of emissions for one species. Compared with (2), the general situation leads us to consider the following model

$$
\frac{d \delta c}{d t}=\mathbf{A} \delta c+B(t) \delta e(t),
$$

where $B(t)$ is an operator transforming the emission state vector into the concentration-state space. Combining with (8), we obtain the extended model

$$
\left(\begin{array}{l}
\delta c(t) \\
\delta e(t)
\end{array}\right)=\left(\begin{array}{cc}
M\left(t, t_{0}\right) \int_{t_{0}}^{t} M(t, s) B(s) M_{e}\left(s, t_{0}\right) d s \\
0 \\
M_{e}\left(t, t_{0}\right)
\end{array}\right)\left(\begin{array}{c}
\delta c\left(t_{0}\right) \\
\delta e\left(t_{0}\right)
\end{array}\right) .
$$

\section{Appendix B}

In this Appendix, we derive the foundation matrix of DFS based on the Kalman smoother and evaluate the potential improvement of the estimate via SVD.

For the discrete-time linear system (12) with the observation mapping (13) in Section 3, we denote the BLUE of $x\left(t_{i}\right)$ based on $\left\{y\left(t_{0}\right), \cdots, y\left(t_{k}\right)\right\}$ by $\hat{x}\left(t_{i} \mid t_{k}\right), t_{i}, t_{k} \in\left[t_{0}, \cdots, t_{N}\right]$. Especially, the prior estimation, or background state, of $x\left(t_{0}\right)$ is denoted by $\hat{x}\left(t_{0} \mid t_{-1}\right)$. Correspondingly, $P\left(t_{i} \mid t_{k}\right)$ is defined as the error covariance of $\hat{x}\left(t_{i} \mid t_{k}\right), t_{i} \in\left[t_{0}, \cdots, t_{N}\right]$ and $t_{k} \in\left[t_{-1}, \cdots, t_{N}\right]$. It is known that the inverse of the analysis error covariance matrix at initial time, $P^{-1}\left(t_{0} \mid t_{N}\right)$ of a fixed-interval Kalman smoother is the optimal Hessian of the underlying cost function of 4D-Var [30]. Thus, we have

$$
\begin{aligned}
P^{-1}\left(t_{0} \mid t_{N}\right) & =P^{-1}\left(t_{0} \mid t_{-1}\right)+\sum_{i=0}^{N} M^{\top}\left(t_{i}, t_{0}\right) H^{\top}\left(t_{i}\right) R^{-1}\left(t_{i}\right) H\left(t_{i}\right) M\left(t_{i}, t_{0}\right) \\
& =P^{-1}\left(t_{0} \mid t_{-1}\right)+\mathcal{G}^{\top} \mathcal{R}^{-1} \mathcal{G}
\end{aligned}
$$

where

$$
\mathcal{G}=\left(\begin{array}{c}
H\left(t_{0}\right) M\left(t_{0}, t_{0}\right) \\
H\left(t_{1}\right) M\left(t_{1}, t_{0}\right) \\
\vdots \\
H\left(t_{N}\right) M\left(t_{N}, t_{0}\right)
\end{array}\right), \mathcal{R}^{-1}=\left(\begin{array}{cccc}
R^{-1}\left(t_{0}\right) & & & \\
& R^{-1}\left(t_{1}\right) & \\
& & \ddots & \\
& & & R^{-1}\left(t_{N}\right)
\end{array}\right) .
$$

It is clear that (41) comprises the information of the initial condition, model evolution, observation configurations and errors over the entire time interval $\left[t_{0}, \cdots, t_{N}\right]$. At the same time, it is independent of any specific data and state vector, apart from the reference model evolution $M(\cdot, \cdot)$ as well as 
the observation operator $H(\cdot)$. Besides, $\mathcal{G}^{\top} R^{-1} \mathcal{G}$ is the observability Gramian with respect to $\mathcal{R}^{-1}$ in control theory [7]. It represents the observation capacity of the observation networks with respect to the model.

It can be seen now that (41) includes all available information before starting the data assimilation procedure, in order to evaluate the potential improvement of the estimate by the Kalman smoother, we aspire a matrix, which allows us for a direct and normalized comparison between sensitivities to initial values and emission rates. To this end, we consider matrix $\tilde{P}$ with the following form:

$$
\begin{aligned}
\tilde{P} & =P^{-\frac{1}{2}}\left(t_{0} \mid t_{-1}\right)\left(P\left(t_{0} \mid t_{-1}\right)-P\left(t_{0} \mid t_{N}\right)\right) P^{-\frac{1}{2}}\left(t_{0} \mid t_{-1}\right) \\
& =I-P^{-\frac{1}{2}}\left(t_{0} \mid t_{-1}\right) P\left(t_{0} \mid t_{N}\right) P^{-\frac{1}{2}}\left(t_{0} \mid t_{-1}\right),
\end{aligned}
$$

where $I$ is the identity matrix and $P^{\frac{1}{2}}\left(t_{0} \mid t_{-1}\right)$ satisfies $P^{\frac{1}{2}}\left(t_{0} \mid t_{-1}\right) P^{\frac{1}{2}}\left(t_{0} \mid t_{-1}\right)=$ $P\left(t_{0} \mid t_{-1}\right)$.

The matrix $\tilde{P}$ is a normalized matrix of the difference between the background forecast error covariance matrix $P\left(t_{0} \mid t_{-1}\right)$ and the analysis error covariance matrix $P\left(t_{0} \mid t_{N}\right)$, as inferred from the Kalman smoother. It is the foundation matrix to study the DFS of models ([20], [38], [40]), which shows how much the observation networks improve the estimation of model states.

Since $P\left(t_{0} \mid t_{N}\right)$ is unknown prior to the data assimilation procedure, we use (41) to rewrite $\tilde{P}$ as

$$
\begin{aligned}
\tilde{P} & =P^{-\frac{1}{2}}\left(t_{0} \mid t_{-1}\right)\left(P\left(t_{0} \mid t_{-1}\right)-P\left(t_{0} \mid t_{N}\right)\right) P^{-\frac{1}{2}}\left(t_{0} \mid t_{-1}\right) \\
& =I-\left(I+P^{\frac{1}{2}}\left(t_{0} \mid t_{-1}\right) \mathcal{G}^{\top} \mathcal{R}^{-1} \mathcal{G} P^{\frac{1}{2}}\left(t_{0} \mid t_{-1}\right)\right)^{-1} .
\end{aligned}
$$

It is worth noting that in $(44)$

$$
I+P^{\frac{1}{2}}\left(t_{0} \mid t_{-1}\right) \mathcal{G}^{\top} \mathcal{R}^{-1} \mathcal{G} P^{\frac{1}{2}}\left(t_{0} \mid t_{-1}\right)
$$

is always invertible even if the observation Gramian $\mathcal{G}^{\top} \mathcal{G}$ is not full-rank. Thus, $\tilde{P}$ is well-defined for all models with invertible initial covariance and observation systems with invertible error covariances within the assimilation window $t_{0}$ to $t_{N}$. Then, we apply the singular value decomposition to simplify (44)

$$
P^{\frac{1}{2}}\left(t_{0} \mid t_{-1}\right) \mathcal{G}^{\top} \mathcal{R}^{-\frac{1}{2}}=V S U^{\top},
$$

where $V$ and $U$ are unitary matrices consisting of the left and right singular vectors respectively, while $S$ is the rectangular diagonal matrix consisting of the singular values.

Then, (44) can be simplified as

$$
\tilde{P}=I-\left(I+V S S^{\top} V^{\top}\right)^{-1}=V\left(I-\left(I+S S^{\top}\right)^{-1}\right) V^{\top}=\sum_{i=1}^{r} \frac{s_{i}^{2}}{1+s_{i}^{2}} v_{i} v_{i}^{\top}
$$

where $r$ is the rank of (44) and $v_{i}$ is the $i^{\text {th }}$ left singular vector in $V$ related to the singular value $s_{i}$, which is the $i^{\text {th }}$ element on the diagonal of $S$. 


\section{Appendix C}

In this appendix, we investigate the ensemble version of Appendix B. For the discrete-time system (12), we denote the ensemble samples of $\hat{x}\left(t_{i} \mid t_{j}\right)$ by $\hat{x}_{k}\left(t_{i} \mid t_{j}\right), i, j=1, \cdots, N, k=1, \cdots, q$, where $q$ is the number of ensemble members.

Correspondingly, the ensemble means of $\hat{x}\left(t_{i} \mid t_{j}\right)$ is given by

$$
\bar{x}\left(t_{i} \mid t_{j}\right)=\frac{1}{q} X\left(t_{i} \mid t_{j}\right) \mathbf{1}_{q \times 1},
$$

where $X\left(t_{i} \mid t_{j}\right)=\left(\hat{x}_{1}\left(t_{i} \mid t_{j}\right), \hat{x}_{2}\left(t_{i} \mid t_{j}\right), \cdots, \hat{x}_{q}\left(t_{i} \mid t_{j}\right)\right)$ is the $n \times q$ ensemble matrix, $\mathbf{1}_{i \times j}$ is a $i \times j$ matrix of which each element is equal to 1 .

We calculate the ensemble forecast and analysis covariances as

$$
\bar{P}\left(t_{i} \mid t_{j}\right)=\frac{1}{q-1} \tilde{X}\left(t_{i} \mid t_{j}\right) \tilde{X}^{\top}\left(t_{i} \mid t_{j}\right)
$$

where $\tilde{X}\left(t_{i} \mid t_{j}\right)=X\left(t_{i} \mid t_{j}\right)-\frac{1}{q} X\left(t_{i} \mid t_{j}\right) \mathbf{1}_{q \times q}$ is the related perturbation matrix. We define the ensemble observation configurations in the entire assimilation window as

$$
y_{k}^{f}=\mathcal{G} \hat{x}_{k}\left(t_{0} \mid t_{-1}\right), \quad k=1, \cdots, q .
$$

Further, the ensemble mean and the forecasting error covariance matrix of the ensemble observation configurations are given by

$$
\bar{y}^{f}=\frac{1}{q} \sum_{k=1}^{q} y_{k}^{f}, \quad \bar{P}_{y y}^{f}=\frac{1}{q-1} \sum_{k=1}^{q}\left(\hat{y}_{k}^{f}-\bar{y}^{f}\right)\left(\hat{y}_{k}^{f}-\bar{y}^{f}\right)^{\top}=\mathcal{G} \bar{P}\left(t_{0} \mid t_{-1}\right) \mathcal{G}^{\top} .
$$

Similarly, we denote the ensemble covariance between the initial states and the forecasting observations by

$$
\bar{P}_{x y}^{f}=\frac{1}{q-1} \sum_{k=1}^{q}\left(\hat{x}_{k}\left(t_{0} \mid t_{-1}\right)-\bar{x}\left(t_{0} \mid t_{-1}\right)\right)\left(\hat{y}_{k}^{f}-\bar{y}^{f}\right)^{\top}=\bar{P}\left(t_{0} \mid t_{-1}\right) \mathcal{G}^{\top} .
$$

Furthermore, defining the ensemble observations as

$$
\hat{y}_{k}\left(t_{i}\right)=y\left(t_{i}\right)+\nu_{k}\left(t_{i}\right), \quad k=1, \cdots, q, \quad i=1, \cdots, N,
$$

we assume $\bar{\nu}\left(t_{i}\right)=\frac{1}{q} \sum_{k=1}^{q} \nu_{k}\left(t_{i}\right)=0, \bar{R}\left(t_{i}\right)=\frac{1}{q-1} \sum_{k=1}^{q} \nu_{k}\left(t_{i}\right) \nu_{k}^{\top}\left(t_{i}\right)$ and $\overline{\mathcal{R}}^{-1}$ is the block diagonal matrix with the diagonal $\left(\bar{R}^{-1}\left(t_{0}\right), \cdots, \bar{R}^{-1}\left(t_{N}\right)\right)$.

It is shown by [18] that the ensemble forecast and analysis covariances have the same form as the covariances in the standard Kalman filter. However, the ensemble size $q$ is significantly smaller than the dimension of the model $n$ in practical applications. As a result, the initial ensemble covariance $\bar{P}\left(t_{0} \mid t_{-1}\right)$ is not invertible. In this case, the pseudo inverse is a widely used alternative of 
the inverse of a matrix, due to its optimal uniqueness properties. We denote the pseudo inverse of a matrix $A$ by $A^{\dagger}$. Then, for the initial ensemble covariance

$$
\bar{P}\left(t_{0} \mid t_{-1}\right)=\frac{1}{q-1} \tilde{X}\left(t_{0} \mid t_{-1}\right) \tilde{X}^{\top}\left(t_{0} \mid t_{-1}\right)
$$

we apply the singular value decomposition to

$$
\frac{1}{\sqrt{q-1}} \tilde{X}\left(t_{0} \mid t_{-1}\right)=V_{0} S_{0} U_{0}^{\top}
$$

where $V_{0} \in \mathbf{R}^{n \times n}$ and $U_{0} \in \mathbf{R}^{q \times q}$ consist of the left and right singular vectors respectively, and $S_{0} \in \mathbf{R}^{n \times q}$ is a rectangular diagonal matrix with singular values $\left\{s_{0 i} \mid s_{0 i} \geqslant 0\right\}_{i=1}^{q}$ on its diagonal. Thus,

$$
\bar{P}\left(t_{0} \mid t_{-1}\right)=V_{0} S_{0} U_{0}^{\top} U_{0} S_{0}^{\top} V_{0}^{\top}=V_{0} S_{0} S_{0}^{\top} V_{0}^{\top}=V_{0} \hat{S}_{0}^{2} V_{0}^{\top},
$$

where $\hat{S}_{0}^{2}=S_{0} S_{0}^{\top} \in \mathbf{R}^{n \times n}$ is a block diagonal matrix with the diagonal

$$
\left(s_{01}^{2}, \cdots, s_{0 r_{0}}^{2}, 0_{1 \times\left(n-r_{0}\right)}\right),
$$

with $r_{0}$ being the rank of $S_{0}$. Hence, we find a pseudo inverse

$$
\bar{P}^{\dagger \frac{1}{2}}\left(t_{0} \mid t_{-1}\right)=V_{0} \hat{S}_{0}^{\dagger} V_{0}^{\top},
$$

where $\hat{S}_{0}^{\dagger}$ is the pseudo inverse of $\hat{S}_{0}$ with the diagonal

$$
\left(1 / s_{01}, \cdots, 1 / s_{0 r_{0}}, 0_{1 \times\left(n-r_{0}\right)}\right) .
$$

Analog to (43), we define $\bar{P}$ as

$$
\bar{P}=\bar{P}^{\dagger \frac{1}{2}}\left(t_{0} \mid t_{-1}\right)\left(\bar{P}\left(t_{0} \mid t_{-1}\right)-\bar{P}\left(t_{0} \mid t_{N}\right)\right) \bar{P}^{\dagger \frac{1}{2}}\left(t_{0} \mid t_{-1}\right) .
$$

Likewise, corresponding to (13), we present the observation system in the entire time interval as

$$
y=\mathcal{G} x\left(t_{0}\right)+\nu,
$$

where $y=\left(y^{\top}\left(t_{0}\right), \cdots, y^{\top}\left(t_{N}\right)\right)^{\top}, \nu=\left(\nu^{\top}\left(t_{0}\right), \cdots, \nu^{\top}\left(t_{N}\right)\right)^{\top}$ and $\mathcal{G}$ as the observation configuration for $x\left(t_{0}\right)$. Then, for the analysis error covariance matrix, we obtain

$$
\begin{aligned}
& \bar{P}\left(t_{0} \mid t_{N}\right) \\
= & \bar{P}\left(t_{0} \mid t_{-1}\right)-\bar{P}\left(t_{0} \mid t_{-1}\right) \mathcal{G}^{\top}\left(\mathcal{G} \bar{P}\left(t_{0} \mid t_{-1}\right) \mathcal{G}^{\top}+\mathcal{R}\right)^{-1} \mathcal{G} \bar{P}\left(t_{0} \mid t_{-1}\right) \\
= & \bar{P}\left(t_{0} \mid t_{-1}\right)-\bar{P}\left(t_{0} \mid t_{-1}\right) \mathcal{G}^{\top} \mathcal{R}^{-\frac{1}{2}}\left(I+\mathcal{R}^{-\frac{1}{2}} \mathcal{G} \bar{P}\left(t_{0} \mid t_{-1}\right) \mathcal{G}^{\top} \mathcal{R}^{-\frac{1}{2}}\right)^{-1} \mathcal{R}^{-\frac{1}{2}} \mathcal{G} \bar{P}\left(t_{0} \mid t_{-1}\right) \\
= & \bar{P}\left(t_{0} \mid t_{-1}\right)-\bar{P}_{x y}^{f} \mathcal{R}^{-\frac{1}{2}}\left(I+\mathcal{R}^{-\frac{1}{2}} \bar{P}_{y y}^{f} \mathcal{R}^{-\frac{1}{2}}\right)^{-1} \mathcal{R}^{-\frac{1}{2}}\left(\bar{P}_{x y}^{f}\right)^{\top} .
\end{aligned}
$$

Further, analog to (47), we obtain

$$
\begin{aligned}
\bar{P} & =\bar{P}^{\dagger \frac{1}{2}}\left(t_{0} \mid t_{-1}\right)\left(\bar{P}\left(t_{0} \mid t_{-1}\right)-\bar{P}\left(t_{0} \mid t_{N}\right)\right) \bar{P}^{\dagger \frac{1}{2}}\left(t_{0} \mid t_{-1}\right) \\
& =\bar{P}^{\dagger \frac{1}{2}}\left(t_{0} \mid t_{-1}\right) \bar{P}_{x y}^{f} \mathcal{R}^{-\frac{1}{2}}\left(I+\mathcal{R}^{-\frac{1}{2}} \bar{P}_{y y}^{f} \mathcal{R}^{-\frac{1}{2}}\right)^{-1} \mathcal{R}^{-\frac{1}{2}}\left(\bar{P}_{x y}^{f}\right)^{\top} \bar{P}^{\dagger \frac{1}{2}}\left(t_{0} \mid t_{-1}\right) .
\end{aligned}
$$


Let $\sum_{i=1}^{N} m\left(t_{i}\right)=m$ be the number of observations available within the assimilation window. To proceed with (61), we apply again the singular value decomposition for the following matrix

$$
\bar{P}^{\dagger \frac{1}{2}}\left(t_{0} \mid t_{-1}\right) \bar{P}_{x y}^{f} \mathcal{R}^{-\frac{1}{2}}=\bar{V} \bar{S} \bar{U}^{\top} \in \mathbf{R}^{n \times m},
$$

where $\bar{U} \in \mathbf{R}^{m \times m}$ and $\bar{V} \in \mathbf{R}^{n \times n}$ consists of the right and left singular vectors of $\bar{P}^{\dagger \frac{1}{2}}\left(t_{0} \mid t_{-1}\right) \bar{P}_{x y}^{f} \mathcal{R}^{-\frac{1}{2}}$, respectively. $S \in \mathbf{R}^{n \times m}$ consists of the singular values on its diagonal.

We denote the rank of (62) by $r$. Then, we rewrite $\bar{P}$ as

$$
\begin{aligned}
\bar{P} & =\bar{P}^{\dagger \frac{1}{2}}\left(t_{0} \mid t_{-1}\right)\left(\bar{P}\left(t_{0} \mid t_{-1}\right)-\bar{P}\left(t_{0} \mid t_{N}\right)\right) \bar{P}^{\dagger \frac{1}{2}}\left(t_{0} \mid t_{-1}\right) \\
& =\bar{V} \bar{S}^{\top} \bar{U}^{\top}\left(\bar{U} \bar{U}^{\top}+\bar{U}\left(\bar{S} \bar{S}^{\top}\right) \bar{U}^{\top}\right)^{-1} \bar{U} \bar{S} \bar{V}^{\top} \\
& =\bar{V} \bar{S}^{\top}\left(I+\bar{S}^{\top} \bar{S}\right)^{-1} \bar{S} \bar{V}^{\top} \\
& =\sum_{i=1}^{r} \frac{\bar{s}_{i}^{2}}{1+\bar{s}_{i}^{2}} \bar{v}_{i} \bar{v}_{i}^{\top} .
\end{aligned}
$$

where $r$ is the rank of $\bar{P}$ as well, and $\bar{v}_{i}$ is the $i^{\text {th }}$ left singular vector in $V$ related to the singular value $\bar{s}_{i}$, which is the $i^{\text {th }}$ element on the diagonal of $S$.

\section{Appendix D}

In this appendix, we will introduce the singular vector approach to identify the sensitive directions of initial values and emission rates to the observation networks.

Similar to [31], we define the magnitude of the perturbation of the initial state by the norm in the state space with respect to a positive definite matrix $W_{0}$, typically the forecast error covariance matrix.

$$
\left\|\delta x\left(t_{0}\right)\right\|_{W_{0}}^{2}=\left\langle\delta x\left(t_{0}\right), W_{0} \delta x\left(t_{0}\right)\right\rangle .
$$

Likewise, we define the magnitude of the related observations perturbation in the time interval $\left[t_{0}, \cdots, t_{N}\right]$ by the norm with respect to a sequence of positive definite matrices $\left\{W\left(t_{k}\right)\right\}_{k=1}^{N}$

$$
\left\|\delta y_{c}\right\|_{\left\{W\left(t_{k}\right)\right\}}^{2}=\sum_{k=0}^{N}\left\langle\delta y_{c}\left(t_{k}\right), W\left(t_{k}\right) \delta y_{c}\left(t_{k}\right)\right\rangle
$$

where $\delta y_{c}=\left(\delta y_{c}^{\top}\left(t_{0}\right), \cdots, \delta y_{c}^{\top}\left(t_{N}\right)\right)^{\top}$.

In order to find the direction of observation configuration which minimizes the perturbation of the initial states, the ratio

$$
\frac{\left\|\delta x\left(t_{0}\right)\right\|_{W_{0}}^{2}}{\left\|\delta y_{c}\right\|_{\left\{W\left(t_{k}\right)\right\}}^{2}}, \quad \delta y \neq 0_{m \times 1} .
$$


must be minimized. It is equivalent to maximize the ratio of the magnitude of observation perturbation and the initial perturbation

$$
\frac{\left\|\delta y_{c}\right\|_{\left\{W\left(t_{k}\right)\right\}}^{2}}{\left\|\delta x\left(t_{0}\right)\right\|_{W_{0}}^{2}}, \quad \delta x\left(t_{0}\right) \neq 0_{n \times 1} .
$$

Thus, we define the measure the perturbation growth as

$$
\begin{aligned}
& g^{2}=\frac{\left\|\delta y_{c}\right\|_{\left\{W\left(t_{k}\right)\right\}}^{2}}{\left\|\delta x\left(t_{0}\right)\right\|_{W_{0}}^{2}} \\
= & \sum_{k=0}^{N} \frac{\left\langle\delta y_{c}\left(t_{k}\right), W\left(t_{k}\right) \delta y_{c}\left(t_{k}\right)\right\rangle}{\left\langle\delta x\left(t_{0}\right), W_{0} \delta x\left(t_{0}\right)\right\rangle} \\
= & \sum_{k=0}^{N} \frac{\left\langle H\left(t_{k}\right) \delta x\left(t_{k}\right), W\left(t_{k}\right) H\left(t_{k}\right) \delta x\left(t_{k}\right)\right\rangle}{\left\langle\delta x\left(t_{0}\right), W_{0} \delta x\left(t_{0}\right)\right\rangle} \\
= & \sum_{k=0}^{N} \frac{\left\langle\delta x\left(t_{k}\right), H\left(t_{k}\right)^{\top} W\left(t_{k}\right) H\left(t_{k}\right) \delta x\left(t_{k}\right)\right\rangle}{\left\langle\delta x\left(t_{0}\right), W_{0} \delta x\left(t_{0}\right)\right\rangle} \\
= & \sum_{k=0}^{N} \frac{\left\langle\delta x\left(t_{0}\right), M\left(t_{k}, t_{0}\right)^{\top} H\left(t_{k}\right) W\left(t_{k}\right) H\left(t_{k}\right) M\left(t_{k}, t_{0}\right) \delta x\left(t_{0}\right)\right\rangle}{\left\langle\delta x\left(t_{0}\right), W_{0} \delta x\left(t_{0}\right)\right\rangle} \\
= & \frac{\left\langle\delta x\left(t_{0}\right), \sum_{k=0}^{N} M\left(t_{k}, t_{0}\right)^{\top} H\left(t_{k}\right)^{\top} W\left(t_{k}\right) H\left(t_{k}\right) M\left(t_{k}, t_{0}\right) \delta x\left(t_{0}\right)\right\rangle}{\left\langle\delta x\left(t_{0}\right), W_{0} \delta x\left(t_{0}\right)\right\rangle} \\
= & \frac{\left\langle\delta x\left(t_{0}\right), \mathcal{G}^{\top} \mathcal{W G} \delta x\left(t_{0}\right)\right\rangle}{\left\langle\delta x\left(t_{0}\right), W_{0} \delta x\left(t_{0}\right)\right\rangle}, \quad \delta x\left(t_{0}\right) \neq 0,
\end{aligned}
$$

where $\mathcal{G}$ has the same definition as given in Section 3 , and

$$
\mathcal{W}=\left(\begin{array}{ccc}
W\left(t_{0}\right) & & \\
& \ddots & \\
& & W\left(t_{N}\right)
\end{array}\right) .
$$

We arrive at the eigenvalue problems [31]

$$
W_{0}^{-\frac{1}{2}} \mathcal{G}^{\top} \mathcal{W} \mathcal{G} W_{0}^{-\frac{1}{2}} v_{k}=s_{k}^{2} v_{k}, \quad W^{\frac{1}{2}} \mathcal{G} W_{0}^{-1} \mathcal{G}^{\top} W^{\frac{1}{2}} u_{k}=s_{k}^{2} u_{k},
$$

where $s_{1} \geqslant s_{2} \geqslant \cdots \geqslant s_{n} \geqslant 0$ are singular values, $\left\{v_{k}\right\}_{k=1}^{n}$ and $\left\{u_{k}\right\}_{k=1}^{n}$ are the corresponding orthogonal singular vectors. Then, $\max _{\delta x\left(t_{0}\right) \neq 0} g^{2}=s_{1}^{2}$.

Especially, if the perturbation norms are provided by the choice $W_{0}=$ $P^{-1}\left(t_{0} \mid t_{-1}\right)$ and $\mathcal{W}=\mathcal{R}^{-1}$,

$$
g^{2}=\frac{\left\langle\delta x\left(t_{0}\right), \mathcal{G}^{\top} \mathcal{R}^{-1} \mathcal{G} \delta x\left(t_{0}\right)\right\rangle}{\left\langle\delta x\left(t_{0}\right), P^{-1}\left(t_{0} \mid t_{-1}\right) \delta x\left(t_{0}\right)\right\rangle}, \quad \delta x\left(t_{0}\right) \neq 0 .
$$

Correspondingly, we consider the singular value decomposition of the following matrices

$$
P^{\frac{1}{2}}\left(t_{0} \mid t_{-1}\right) \mathcal{G}^{\top} \mathcal{R}^{-1} \mathcal{G} P^{\frac{1}{2}}\left(t_{0} \mid t_{-1}\right) v_{k}=s_{k}^{2} v_{k}
$$




$$
\mathcal{R}^{-\frac{1}{2}} \mathcal{G} P\left(t_{0} \mid t_{-1}\right) \mathcal{G}^{\top} \mathcal{R}^{-\frac{1}{2}} u_{k}=s_{k}^{2} u_{k}, \quad k=1, \cdots, n
$$

where $s_{k}, v_{k}$ and $u_{k}, k=1, \ldots, n$ denotes the singular values and corresponding singular vectors to simplify the notation. At the same time, it can be seen that the above singular value decomposition problem coincides with (46) in Section 3.

\section{Acknowledgements}

This study was supported by HITEC (Helmholtz Interdisciplinary Doctoral Training in Energy and Climate Research). HITEC is the Graduate School of Forschungszentrum Jlich and the five partner universities Aachen, Bochum, Cologne, Düsseldorf and Wuppertal focusing on energy and climate research. Technical and computational support was provided by the Jülich Supercomputer Centre (JSC) of the Research Centre Jülich. A large and central part of the case studies has been computed on JSC supercomputer JURECA. Access given to these computational resources is highly appreciated.

\section{References}

1. Anderson, J. L.: An ensemble adjustment Kalman filter for data assimilation, Monthly weather review, 129, 2884-2903, 2001.

2. Bellsky, T., Kostelich, E. J. and Mahalov, A.: Kalman filter data assimilation: targeting observations and parameter estimation, Chaos, 24(2):024406, doi: 10.1063/1.4871916, 2014 .

3. Berliner, L. M., Lu, Z., and Snyder, C.: Statistical design for adaptive weather observations, J. Atmos. Sci., 56, 2536-2552, 1998.

4. Bishop, C. H. and Toth. Z: Ensemble transformation and adaptive observations, J. Atmos. Sci. 56, 1748-1765, 1999.

5. Bocquet, M., Sakov, P.: Joint state and parameter estimation with an iterative ensemble Kalman smoother, Nonlinear proc geoph., 20, 803-818, 2013.

6. Bousserez, N., Henze, D. K.: Optimal and scalable methods to approximate the solutions of large-scale Bayesian problems: theory and application to atmospheric inversion and data assimilation, Q. J. R. Meteorol. Soc., 144, 365-390, 2018.

7. Brockett R. W.: Finite dimensional linear systems, John Wiley and Sons, Inc, 1970.

8. Buizza, R. and Montani, A.: Targeting observations using singular vectors, J. Atmos. Sci, 56, 2965-2985, 1999.

9. Buizza, R. and Palmer, T. N.: The singular-vector structure of the atmospheric global circulation, J. Atom. Sci., 52, 1434-1456, 1995.

10. Cardinali, C., Pezzulli, S. and Andersson, E.: Influence-matrix diagnostic of a data assimilation system, Q. J. R. Meteorol. Soc., 130, 2767-2786, 2004.

11. Cioaca A. and Sandu A.: Low-rank approximations for computing observation impact in 4D-Var data assimilation, Compu. \& Math. with Appli., 67(12), 2112-2126, 2014.

12. Cioaca, A. and Sandu, A.: An optimization framework to improve 4D-Var data assimilation system performance, J. Compu. Phy., 275, 377-389, 2014.

13. Daescu, D. and Navon, I. M.: Adaptive observations in the context for 4D-Var data assimilation, Meteorol. Atmos. Phys., 55, 205-236, 2004.

14. Daley, R.: Atmospheric data analysis, Cambridge University Press, 1991.

15. Elbern, H. and Schmidt, H.: A four-dimensional variational chemistry data assimilation scheme for Eulerian chemistry transport modeling, J. Geophys. Res., 104, 583-598, 1999. 
16. Elbern, H., Schmidt, H., Talagrand, O. and Ebel, A.: 4D-variational data assimilation with an adjoint air quality model for emission analysis, Environ. modell. softw., 15, 539548, 2000.

17. Elbern, H., Strunk, A., Schmidt, H. and Talagrand, O.: Emission rate and chemical state estimation by 4-dimension variational inversion, Atmos. Chem. Phys., 7, 3749-3769, 2007.

18. Evensen, G.: Data assimilation: The ensemble Kalman filter, 2th Edition, Springer, Norway, 2009.

19. Eyre, J. R.: The Information Content of Data From Satellite Sounding Systems: A Simulation Study, Quart. J. Roy. Meteor. Soc., 116, 401-434, 1990.

20. Fisher, M.: Estimation of Entropy reduction and Degrees of freedom for signal for large variational analysis systems, ECMWF Technical Memorandum,397, 2003.

21. A. Gelb, (ed.): Applied Optimal Estimation, Cambridge, Mass.: M.I.T. Press, 1974.

22. Goris, N. and Elbern, H.: Singular vector decomposition for sensitivity analyses of tropospheric chemical scenarios, Atmos. Chem. Phys., 13, 5063-5087, 2013.

23. Goris, N. and Elbern, H.: Singular vector based targeted observations of chemical constituents: description and first application of EURAD-IM-SVA, Geosci. Model Dev, 8, 3929-3945, 2015.

24. Higham, N. J.: Accuracy and stability of numerical algorithms, Second edition, SIAM, 2002 .

25. Johnson, C.: Information content of observations in variational data assimilation, $\mathrm{Ph}$. D thesis, 2003.

26. Kang, W. and $\mathrm{Xu}$, L.: Optimal placement of mobile sensors for data assimilations, Tellus A, 64, 2012 .

27. Khattatov, B. B., Gille, J., Lyjak, L., Brasseur, G., Dvortsov, V., Roche, A. and Waters, J.: Assimilation of photochemically active species and a case analysis of UARS data, J. Geophys. Res., 22, 18715-18738, 1999.

28. Langland, R. H., Gelaro, R., Rohaly, G. D., and Shapiro, M. A.: Targeted observations in FASTEX: Adjoint based targeting procedures and data impact experiments in IOP17 and IOP18, Q. J. R. Meteorolog. Soc. 125, 3241-3270, 1999.

29. Li, H., Kalnay, E., Miyoshi, T., Danforth, C. M.: Accounting for model errors in ensemble data assimilation, Mon. Weather Rev., 137, 3407-3419, 2009.

30. Li, Z., Navon, I. M.: Optimality of variational data assimilation and its relationship with the Kalman filter and smoother, Q. J. R. Meteorol. Soc., 127, 661-683, 2001.

31. Liao, W., Sandu, A., Carmichael , G. R. and Chai, T.: Singular vector analysis for atmospheric chemical transport models, Month. Weather Rev., 134, 2443-2465, 2006.

32. Lorenz, E.N.: A study of the predictability of a 28 variable atmospheric model, Tellus, $17,321-333,1965$.

33. Martynenko, D., Holzer-Popp, T., Elbern, H. and Schroedter-Homscheidt, M.: Understanding the aerosol information content in multi-spectral reflectance measurements using a synergetic retrieval algorithm, Atmos. Meas. Tech., 3, 2579-2602, 2010.

34. Miyazaki, K., Eskes, H. J., Sudo, K., Takigawa, M., vanWeele, M., and Boersma, K. F.: Simultaneous assimilation of satellite NO2, O-3, CO, and HNO3 data for the analysis of tropospheric chemical composition and emissions, Atmos. Chem. Phys., 12, 9545-9579, 2012 .

35. Navon, I. M.: Practical and theoretical aspects of adjoint parameter estimation and identifiability in meteorology and oceanography, Dyn. Atmos. Oceans, 27, 55-79, 1997.

36. Peters, W., Miller, J. B., Whitaker, J., Denning, A. S., Hirsch, A., Krol, M. C.,Zupanski, D., Bruhwiler, L. and Tans, P. P.: An ensemble data assimilation system to estimate $\mathrm{CO}_{2}$ surface fluxes from atmospheric trace gas observations, J. Geophys. Res., 110(D24), 2005

37. Rabier, F., Fourrié, N., Chafaï, D., and Prunet, P.: Channel selection methods for infrared atmospheric sounding interferometer radiances. Quart. J. Roy. Meteor. Soc., 128, 1011-1027, 2002

38. Rodgers, C. D.: Inverse methods for atmospheric sounding: theory and practice (Series on atmospheric, oceanic and planetary physics-Vol.2, World scientific, Singapore, 2000.

39. Sandu, A., Cioaca, A. and Rao, V.: Dynamic sensor network configuration in InfoSymbiotic systems using model singular vectors, Procedia Computer Science, 18, 1909-1918, 2013. 
40. Singh, K., Sandu, A., Jardak, M., Bowman, K.W. and Lee, M.: A practical method to estimate information content in the context of $4 \mathrm{D}$-Var data assimilation, SIAM/ASA J. Uncertainty quantification, 1(1), 106-138, 2013.

41. Smith, P. J., Thornhill, G. D., Dance, S. L., Lawless, A. S., Mason, D. C. and Nichols, N. K.: Data assimilation for state and parameter estimation: application to morphodynamic modeling, Q. J. R. Meteorol. Soc., 139, 314-327, 2013.

42. Spantini, A., Solonen, A., Cui, T., Martin, J., Tenorio, L., Marzouk, Y.: Optimal lowrank approximations of Bayesian linear inverse problems, SIAM J. Sci. Comput., 37, A2451A2487, 2015.

43. Szunyogh, I., Toth, Z., Emanuel, K. A., Bishop, C.H., Woolen, J., Marchok, T., Morss, R., and Snyder, C.: Ensemble based targeting experiments during FASTEX: The impact of dropsonde data from the Lear jet, Q. J. R. Meteorolog. Soc. 125, 3189-3218, 1999.

44. Tang, X., Zhu, J., Wang, Z. F., Wang, M., Gbaguidi, A., Li, J., Shao, M., Tang, G. Q., and Ji, D. S.: Inversion of CO emissions over Beijing and its surrounding areas with ensemble Kalman filter, Atmos. Environment, 81, 676-686, 2013.

45. Winiarek, V., Bocquet, M., Duhanyan, N., Roustan, Y., Saunier, O., and Mathieu, A.: Estimation of the caesium-137 source term from the Fukushima Daiichi nuclear power plant using a consistent joint assimilation of air concentration and deposition observations, Atmos. Environment, 82, 268-279, 2014.

46. $\mathrm{Wu}, \mathrm{X}$., Jacob, B. and Elbern, H.: Optimal control and observation locations for timevarying systems on a finite-time horizon, SIAM J. Control Optim., 2016.

47. Woodbury, M. A.: Inverting modified matrices, Memorandum Rept. 42, Statistical Research Group, Princeton University, Princeton, NJ, 1950.

48. Yanenko, N. N.: The method of fractional steps: solution of problems of mathematical physics in several variables, Springer, Berlin, Heidelburg and New York, 1971

49. Zupanski, D., Hou, Y. A., Zhang, Q. S., Zupanski, M., Kummerow, D. C., Cheung, H. S.: Applications of information theory in ensemble data assimilation, Q. J. R. Meteorol. Soc., 133, 1533-1545, 2007. 